\title{
Maximal regularity in continuous interpolation spaces and quasilinear parabolic equations
}

\author{
PHILIPPE ClÉMENT AND GIERI SIMONETT
}

Abstract. In this paper we establish a geometric theory for abstract quasilinear parabolic equations. In particular, we study existence, uniqueness, and continuous dependence of solutions. Moreover, we give conditions for global existence and establish smoothness properties of solutions. The results are based on maximal regularity estimates in continuous interpolation spaces. An important new ingredient is that we are able to show that quasilinear parabolic evolution equations generate a smooth semiflow on the trace spaces associated with maximal regularity, which are the natural phase spaces in this framework.

\section{Introduction}

In this paper we consider the following abstract quasilinear parabolic evolution equation

$$
\left\{\begin{array}{l}
\dot{u}+A(u) u=f(u), \\
u(0)=x .
\end{array}\right.
$$

Throughout we shall assume that $(A, f)$ is a mapping from $V$ into $\mathcal{H}\left(E_{1}, E_{0}\right) \times E_{0}$. Here, $E_{0}$ and $E_{1}$ are two given (real or complex) Banach spaces such that $E_{1}$ is densely embedded in $E_{0}$, and $V$ is a subset of $E_{0}$. Moreover, $\mathcal{H}\left(E_{1}, E_{0}\right)$ denotes the set of all bounded linear operators $B \in \mathcal{L}\left(E_{1}, E_{0}\right)$ such that $-B$ is the infinitesimal generator of a strongly continuous analytic semigroup on $E_{0}$.

Abstract quasilinear parabolic problems have been studied by many authors, including $[1,2,3,4,6,9,10,11,16,18,19]$, and different approaches have been devised to establish the existence of solutions. One approach is based on the concept of parabolic evolution operators and has in particular been used in [18] and in [1, 2, 3]. Another approach relies on the notion of maximal regularity and has for instance been applied in $[4,6,16]$.

The most general and flexible results for Problem (1.1) have been obtained in [2], based on a careful and detailed analysis of parabolic evolution operators on interpolation spaces. The method based on maximal regularity has the drawback of requiring stronger assumptions on the geometry of the spaces $E_{0}$ and $E_{1}$, but has the advantage that one can resort to the Implicit

Received August 10, 2000; accepted September 20, 2000.

2000 Mathematics Subject Classification: 35C90.

Key words: Quasilinear parabolic equitations, maximal regularity, interpolation spaces.

The research of the second author has been partially supported by NSF Grant DMS-9801337. 
Function Theorem to establish additional properties of solutions. Moreover, it provides a natural setting for studying further geometric properties for the semiflow generated by (1.1). In fact, all results known to us concerning center manifolds for quasilinear (and even fully nonlinear) parabolic equations are tied to maximal regularity $[8,13,15,17]$.

If the mapping $(A, f)$ is assumed to be continuously differentiable, then Problem (1.1) can be treated within the theory of fully nonlinear equations developed in [7]. However, since this approach does not take advantage of the quasilinear structure it does not provide the smoothing property of solutions which is an important feature of quasilinear parabolic evolution equations.

In [4], the maximal regularity results of [7] were extended to include functions which admit a prescribed singularity at $t=0$. This extension, in turn, allows to take advantage of the quasilinear structure of (1.1) and to establish the smoothing property, as was shown in [4].

In $[15,16]$ the results of $[4]$ were refined and the continuous and smooth dependence of solutions to (1.1) was established. Moreover, existence and exponential attractivity of center manifolds under appropriate assumptions was proved in [15, 17]. In particular, it was shown that the center manifolds attract solutions in the stronger norm of $E_{1}$ for solutions which start out in interpolation spaces between $E_{1}$ and $E_{0}$, thus taking advantage of the smoothing property. This effect has important consequences for applications and cannot be observed in the approach of [8].

In this paper we establish a geometric theory for the quasilinear parabolic equation (1.1). An important new ingredient is that we are able to show that (1.1) generates a (Lipschitz continuous or smooth) semiflow on the trace spaces associated with maximal regularity, which are the natural phase spaces in this framework. This provides a considerable improvement on the results obtained in $[4,15,16]$. A more detailed discussion is given in Remarks 5.2 and Remarks 6.2.

The paper is organized as follows. In Section 2 we state the results concerning maximal regularity for the linear theory which are used in the next sections. Local existence, uniqueness and continuous dependence of solutions to (1.1) is established in Section 3, Theorem 3.1. In Section 4, we investigate global existence and the main result is contained in Theorem 4.1. In Section 5 we show that Problem (1.1) generates a locally Lipschitz continuous semiflow on the trace spaces associated with maximal regularity. Finally, in Section 6 we establish smoothness properties of solutions, relying on the Implicit Function Theorem.

NOTATIONS. In the sequel we shall use the following notations. If $E$ and $F$ are two Banach spaces we write $E \hookrightarrow F$ if $E$ is continuously embedded in $F$, and we write $E \stackrel{d}{\hookrightarrow} F$ if $E$ is, in addition, dense in $F$. If $X$ and $Y$ are metric spaces and $f$ is a mapping from $X$ into $Y$ we say that $f$ is locally Lipschitz continuous, and we use the notation $f \in C^{1-}(X, Y)$, if every point $x \in X$ has a neighborhood $U$ such that $\left.f\right|_{U}$ is (globally) Lipschitz continuous. 


\section{Function spaces and maximal regularity}

In the following we assume that $\mu \in(0,1]$, that $E$ is a (real or complex) Banach space, and that $J=[0, T]$ for some number $T>0$. We consider functions defined on $\dot{J}:=J \backslash\{0\}$ which have a prescribed singularity at 0 . Let

$$
\begin{aligned}
B U C_{1-\mu}(J, E):= & \left\{u \in C(\dot{J}, E) ;\left[t \mapsto t^{1-\mu} u\right] \in B U C(\dot{J}, E),\right. \\
& \left.\lim _{t \rightarrow 0^{+}} t^{1-\mu}\|u(t)\|=0\right\} \\
\|u\|_{C_{1-\mu}}:= & \sup _{t \in \dot{J}} t^{1-\mu}\|u(t)\|_{E}, \quad \mu \in(0,1) .
\end{aligned}
$$

It is easy to verify that $B U C_{1-\mu}(J, E)$, equipped with the norm $\|\cdot\|_{C_{1-\mu}}$, is a Banach space. Next we introduce the following subspace of $B U C_{1-\mu}(J, E)$

$$
B U C_{1-\mu}^{1}(J, E):=\left\{u \in C^{1}(\dot{J}, E) ; u, \dot{u} \in B U C_{1-\mu}(J, E)\right\} .
$$

Moreover we set

$$
B U C_{0}(J, E):=B U C(J, E), \quad B U C_{0}^{1}(J, E):=B U C^{1}(J, E) .
$$

If $E_{1}$ and $E_{0}$ are two Banach spaces such that $E_{1}$ is continuously embedded in $E_{0}$ we set

$$
\begin{aligned}
& \mathbb{E}_{0}(J):=B U C_{1-\mu}\left(J, E_{0}\right), \quad \mu \in(0,1], \\
& \mathbb{E}_{1}(J):=B U C_{1-\mu}^{1}\left(J, E_{0}\right) \cap B U C_{1-\mu}\left(J, E_{1}\right),
\end{aligned}
$$

where $\mathbb{E}_{1}(J)$ is given the norm

$$
\|u\|_{\mathbb{E}_{1}(J)}:=\sup _{t \in \dot{J}} t^{1-\mu}\left(\|\dot{u}(t)\|_{E_{0}}+\|u(t)\|_{E_{1}}\right)
$$

which turns it into a Banach space. In the following we will use the notation

$$
E_{\theta}:=\left(E_{0}, E_{1}\right)_{\theta}:=\left(E_{0}, E_{1}\right)_{\theta, \infty}^{0}, \quad \theta \in(0,1),
$$

for the continuous interpolation spaces of DaPrato and Grisvard [7], see [3, 4, 13] for more information.

REMARKS 2.1. (a) Let $A \in \mathcal{L}\left(E_{1}, E_{0}\right)$. Then it is easy to see that

$$
\left(\frac{d}{d t}+A\right) \in \mathcal{L}\left(\mathbb{E}_{1}(J), \mathbb{E}_{0}(J)\right)
$$


(b) $E_{1}$ is densely embedded in $E_{1-\mu}$. If $A \in \mathcal{H}\left(E_{1}, E_{0}\right)$ with type $(-A)<0$ and $x \in E_{0}$, then the following characterization is well-known:

$$
\begin{aligned}
x \in\left(E_{0}, E_{1}\right)_{\theta} \Longleftrightarrow \sup _{s>0} s^{1-\theta}\left\|A e^{-s A} x\right\|_{E_{0}}<\infty, \\
\lim _{s \rightarrow 0^{+}} s^{1-\theta}\left\|A e^{-s A} x\right\|_{E_{0}}=0
\end{aligned}
$$

and

$\|x\|_{\theta}:=\sup _{s>0} s^{1-\theta}\left\|A e^{-s A} x\right\|_{E_{0}}$

is an equivalent norm on $E_{\theta}$ for $\theta \in(0,1)$.

(c) Suppose that $A \in \mathcal{H}\left(E_{1}, E_{0}\right)$ and that type $(-A)<0$. Let $x \in E_{\mu}$ be given. Then $\left[t \mapsto e^{-t A} x\right] \in \mathbb{E}_{1}(J)$ and there exists a constant $c>0$ independent of $J$ such that $\left\|e^{-t A} x\right\|_{\mathbb{E}_{1}(J)} \leq c\|x\|_{\mu}, \quad t \in J$.

Proof. (i) Let $\mu \in(0,1)$ be fixed and let $u(t):=e^{-t A} x$ for $t \in J$. It follows from (2.3) that

$$
\sup _{t \in \dot{J}} t^{1-\mu}\left(\|\dot{u}(t)\|_{E_{0}}+\|u(t)\|_{E_{1}}\right) \leq c \sup _{t>0} t^{1-\mu}\left\|A e^{-t A} x\right\|_{E_{0}}=c\|x\|_{\mu}
$$

where $c$ does not depend on $J$.

(ii) It remains to show that $u \in \mathbb{E}_{1}(J)$. Since $E_{1}$ is densely embedded in $E_{\mu}$ we find a sequence $x_{n}$ in $E_{1}$ such that $x_{n} \rightarrow x$ in $E_{\mu}$. Let $u_{n}(t):=e^{-t A} x_{n}$ for $t \in J$. It is easy to see that $u_{n} \in \mathbb{E}_{1}(J)$ and it follows from (i) that

$$
\sup _{t \in \dot{J}} t^{1-\mu}\left(\left\|\dot{u}(t)-\dot{u}_{n}(t)\right\|_{E_{0}}+\left\|u(t)-u_{n}(t)\right\|_{E_{1}}\right) \leq c\left\|x-x_{n}\right\|_{\mu}, \quad n \in \mathbb{N} .
$$

Since $\mathbb{E}_{1}(J)$ is a Banach space we readily conclude that $u \in \mathbb{E}_{1}(J)$.

(iii) The remaining case $\mu=1$ is easy to show.

(d) The mapping $\gamma: \mathbb{E}_{1}(J) \rightarrow E_{0}, \gamma v:=v(0)$, is well-defined, linear and continuous.

Proof. Let $v \in \mathbb{E}_{1}(J)$ be given and let $t$ be a fixed number in $\dot{J}$. Then

$$
v(s)=v(t)-\int_{s}^{t} \dot{v}(\tau) d \tau, \quad 0<s<t,
$$

and we conclude that $v(0)=\lim _{s \rightarrow 0^{+}} v(s)$ exists and admits the representation

$v(0)=v(t)-\int_{0}^{t} \dot{v}(\tau) d \tau$.

The assertion follows now from this representation. 
(e) According to Remark (d), any function $v \in \mathbb{E}_{1}(J)$ has a trace and we can introduce the trace space $\gamma \mathbb{E}_{1}(J)$ of $\mathbb{E}_{1}(J)$,

$$
\begin{aligned}
\gamma \mathbb{E}_{1}(J) & :=\operatorname{im}(\gamma) \\
\|x\|_{\gamma \mathbb{E}_{1}(J)} & :=\inf \left\{\|v\|_{\mathbb{E}_{1}(J)} ; v \in \mathbb{E}_{1}(J), \gamma v=x\right\} .
\end{aligned}
$$

It is easy to verify that $\gamma \mathbb{E}_{1}(J)$ is a Banach space and that $\gamma \in \mathcal{L}\left(\mathbb{E}_{1}(J), \gamma \mathbb{E}_{1}(J)\right)$.

LEMMA 2.2. Suppose that $\mathcal{H}\left(E_{1}, E_{0}\right) \neq \emptyset$. Then

(a) $\gamma \mathbb{E}_{1}(J)=E_{\mu}$.

(b) $\mathbb{E}_{1}(J)=B U C_{1-\mu}^{1}\left(J, E_{0}\right) \cap B U C_{1-\mu}\left(J, E_{1}\right) \hookrightarrow B U C\left(J, E_{\mu}\right)$.

(c) There exists a constant $c>0$ independent of $J$ such that

$$
\|u\|_{B U C\left(J, E_{\mu}\right)} \leq c\|u\|_{\mathbb{E}_{1}(J)}, \quad u \in \mathbb{E}_{1}(J), \quad \gamma u=0,
$$

(d) $\mathbb{E}_{1}(J)=B U C_{1-\mu}^{1}\left(J, E_{0}\right) \cap B U C_{1-\mu}\left(J, E_{1}\right) \hookrightarrow B U C^{\mu-\sigma}\left(J, E_{\sigma}\right), \quad \sigma \in[0, \mu]$.

Proof. (a) For a proof we refer to [3, Theorem III.2.3.1].

(b) This follows from [3, Theorem III.2.3.3].

(c) We choose $A \in \mathcal{H}\left(E_{1}, E_{0}\right)$ with type $(-A)<0$ and equip $E_{\mu}$ with the norm (2.3). Suppose that $u \in \mathbb{E}_{1}(J), \gamma u=0$ and let $t \in J$ be given. If $0<s \leq t$ we obtain

$s^{1-\mu}\left\|A e^{-s A} u(t)\right\|_{E_{0}} \leq c t^{1-\mu}\|u(t)\|_{E_{1}} \leq c\|u\|_{\mathbb{E}_{1}(J)}$.

If $t<s$ then we use the formula $u(t)=\int_{0}^{t} \dot{u}(\tau) d \tau$, see (2.6), to conclude that

$$
s^{1-\mu}\left\|A e^{-s A} u(t)\right\|_{E_{0}} \leq c s^{-\mu} \int_{0}^{t} \tau^{\mu-1} d \tau\|u\|_{\mathbb{E}_{1}(J)} \leq c(\mu)\|u\|_{\mathbb{E}_{1}(J)} .
$$

The assertion is now a consequence of (2.3).

(d) If $\sigma=\mu$ the assertion follows from part (b). Let $\sigma \in[0, \mu)$. It follows from the reiteration theorem for the continuous interpolation method, see [3, Section I.2.8], that $E_{\sigma}=\left(E_{0}, E_{\mu}\right)_{\sigma / \mu}$. Let $u \in \mathbb{E}_{1}(J)$ be given. The interpolation inequality, (2.5) and part (b) yield

$$
\begin{gathered}
\|u(t)-u(s)\|_{E_{\sigma}} \leq c\|u(t)-u(s)\|_{E_{0}}^{1-\sigma / \mu} \\
\|u(t)-u(s)\|_{E_{\mu}}^{\sigma / \mu} \leq c\|u\|_{\mathbb{E}_{1}(J)}|t-s|^{\mu-\sigma}
\end{gathered}
$$

for $s, t \in J$, proving the claim. 
DEFINITION 2.3. (Maximal regularity) Let $E_{1}$ and $E_{0}$ be two Banach spaces such that $E_{1} \hookrightarrow E_{0}$ and suppose that $A \in \mathcal{H}\left(E_{1}, E_{0}\right)$. Moreover, let $J=[0, T]$ for some $T>0$ and let $\mu \in(0,1]$. Then we define

$$
A \in \mathcal{M}_{\mu}\left(E_{1}, E_{0}\right): \Longleftrightarrow\left(\frac{d}{d t}+A, \gamma\right) \in \operatorname{Isom}\left(\mathbb{E}_{1}(J), \mathbb{E}_{0}(J) \times E_{\mu}\right) .
$$

If $A \in \mathcal{M}_{\mu}\left(E_{1}, E_{0}\right)$ then $\left(\mathbb{E}_{0}(J), \mathbb{E}_{1}(J)\right)$ is called a pair of maximal regularity for $A$.

REMARKS 2.4. (a) It follows from Remarks 2.1(a), (e) and Lemma 2.2(a) that

$$
\left(\frac{d}{d t}+A, \gamma\right) \in \mathcal{L}\left(\mathbb{E}_{1}(J), \mathbb{E}_{0}(J) \times E_{\mu}\right)
$$

Moreover, the mapping is injective.

(b) Suppose that $\left(\mathbb{E}_{1}(J), \mathbb{E}_{0}(J)\right)$ is a pair of maximal regularity for $A$ and let $(f, x) \in$ $\mathbb{E}_{1}(J) \times E_{\mu}$ be given. Then

$$
\begin{aligned}
\left(\frac{d}{d t}+A, \gamma\right)^{-1}(f, x) & =e^{-t A} x+\int_{0}^{t} e^{-(t-\tau) A} f(\tau) d \tau \\
& =: e^{-t A} x+\left(K_{A} f\right)(t) .
\end{aligned}
$$

(c) $\left(\mathbb{E}_{0}(J), \mathbb{E}_{1}(J)\right)$ is a pair of maximal regularity for $A$ if and only if

$$
K_{A}\left(B U C_{1-\mu}\left(J, E_{0}\right)\right) \subset B U C_{1-\mu}\left(J, E_{1}\right) .
$$

Proof. (i) Suppose that $\left(\mathbb{E}_{0}(J), \mathbb{E}_{1}(J)\right)$ is a pair of maximal regularity for $A$ and let $f \in B U C_{1-\mu}\left(J, E_{0}\right)$. Then

$$
K_{A} f=\left(\frac{d}{d t}+A, \gamma\right)^{-1}(f, 0) \in \mathbb{E}_{1}(J) \subset B U C_{1-\mu}\left(E_{1}\right)
$$

(ii) Suppose that $K_{A}$ maps $B U C_{1-\mu}\left(J, E_{0}\right)$ into $B U C_{1-\mu}\left(J, E_{1}\right)$. Let $f \in \mathbb{E}_{0}(J)$ be given. Then it follows from [14, Theorem 4.2.4] that $K_{A} f \in C^{1}\left(\dot{J}, E_{0}\right)$ and that

$\frac{d}{d t}\left(K_{A} f\right)=f-A K_{A} f \in B U C_{1-\mu}\left(J, E_{0}\right)$.

We conclude that $K_{A} f \in \mathbb{E}_{1}(J)$ and the assertion follows from (a)-(b), from Remark 2.1(c), and from the open mapping theorem.

(d) Suppose $\left(\mathbb{E}_{0}(J), \mathbb{E}_{1}(J)\right)$ is a pair of maximal regularity for some $A \in \mathcal{H}\left(E_{1}, E_{0}\right)$. Then either $E_{1}=E_{0}$ or $E_{0}$ contains a closed subspace which is isomorphic to the space $c_{0}$ of null sequences. In particular, if $E_{0}$ is reflexive then the set $\mathcal{M}_{\mu}\left(E_{1}, E_{0}\right)$ will be empty. 
Proof. This result follows from [5] and Lemma 2.6.

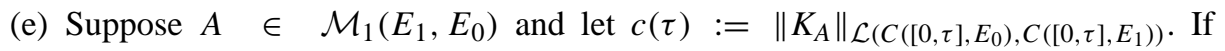
$\lim \sup _{\tau \rightarrow 0} c(\tau)=0$ then $E_{1}=E_{0}$.

Proof. Let $x \in E_{0}$ be given and set $f(t):=e^{-t A} x$. Then $f \in C\left(J, E_{0}\right)$ and

$$
\left(K_{A} f\right)(t)=\int_{0}^{t} e^{-(t-s) A} e^{-s A} x d s=t e^{-t A} x .
$$

If follows from our assumption that

$$
\left\|t A e^{-t A} x\right\|_{E_{0}} \leq\|A\|_{\mathcal{L}\left(E_{1}, E_{0}\right)}\left\|\left(K_{A} f\right)(t)\right\|_{E_{1}} \leq N c(\tau)\|x\|_{E_{0}}
$$

for $t \in[0, \tau]$. This implies $\left\|t A e^{-t A}\right\| \leq N c(\tau)$ for $t \in[0, \tau]$ and the claim follows from [14, Theorem 2.5.3].

(f) We shall now describe a situation which shows that many interesting operators belong to the class $\mathcal{M}_{\mu}\left(E_{1}, E_{0}\right)$.

Assume that $A \in \mathcal{H}\left(E_{1}, E_{0}\right)$ and let

$$
\begin{aligned}
E_{2} & :=E_{2}(A):=\left(\operatorname{dom}\left(A^{2}\right),\|\cdot\|_{E_{2}}\right), \\
\|\cdot\|_{E_{2}} & :=\|\cdot\|_{E_{2}(A)}:=\|A \cdot\|_{E_{1}}+\|\cdot\|_{E_{1}} .
\end{aligned}
$$

Then $\left(E_{2},\|\cdot\|_{E_{2}}\right)$ is a Banach space with $E_{2} \stackrel{d}{\hookrightarrow} E_{1} \stackrel{d}{\hookrightarrow} E_{0}$. We set

$$
E_{\theta}:=\left(E_{0}, E_{1}\right)_{\theta},
$$$$
E_{1+\theta}:=E_{1+\theta}(A):=\left(E_{1}, E_{2}(A)\right)_{\theta}, \quad 0<\theta<1,
$$

$A_{\theta}:=$ the maximal $E_{\theta}$-realization of $A$.

It is well-known that $A_{\theta} \in \mathcal{H}\left(E_{\theta}, E_{1+\theta}\right)$ and it turns out that $A_{\theta} \in \mathcal{M}_{\mu}\left(E_{\theta}, E_{1+\theta}\right)$ for any $\mu \in(0,1]$.

THEOREM. (Da Prato, Grisvard, Angenent) Suppose that $\mu \in(0,1]$ and $\theta \in(0,1)$. Let $J=[0, T]$. Then

$$
\left(\mathbb{E}_{\theta}(J), \mathbb{E}_{1+\theta}(J)\right):=\left(B U C_{1-\mu}\left(J, E_{\theta}\right), B U C_{1-\mu}^{1}\left(J, E_{\theta}\right) \cap B U C_{1-\mu}\left(J, E_{1+\theta}\right)\right)
$$

is a pair of maximal regularity for $A_{\theta}$, that is,

$$
\left(\frac{d}{d t}+A_{\theta}, \gamma\right) \in \operatorname{Isom}\left(\mathbb{E}_{1+\theta}(J), \mathbb{E}_{\theta}(J) \times\left(E_{\theta}, E_{1+\theta}\right)_{\mu}\right)
$$

where $\left(E_{\theta}, E_{1+\theta}\right)_{1}:=E_{1+\theta}$

Proof. This was proved by Da Prato and Grisvard [7] for $\mu=1$, and by Angenent [4] for the general case. We also refer to [3, Theorem III.3.4.1] and [15]. 
LEMMA 2.5. (a) $\mathcal{M}_{\mu}\left(E_{1}, E_{0}\right) \subset \mathcal{H}\left(E_{1}, E_{0}\right)$ is open and

$$
\left[A \mapsto\left(\frac{d}{d t}+A, \gamma\right)^{-1}\right] \in C^{\omega}\left(\mathcal{M}_{\mu}\left(E_{1}, E_{0}\right), \mathcal{L}\left(\mathbb{E}_{0}(J) \times E_{\mu}, \mathbb{E}_{1}(J)\right),\right.
$$

i.e., the mapping is real analytic.

(b) Let $\mathcal{A} \subset \mathcal{M}_{\mu}\left(E_{1}, E_{0}\right)$ be compact. Then there exists a constant $c$ such that

$$
\left\|\left(\frac{d}{d t}+A, \gamma\right)^{-1}\right\|_{\mathcal{L}\left(\mathbb{E}_{0}(J) \times E_{\mu}, \mathbb{E}_{1}(J)\right)} \leq c, \quad A \in \mathcal{A} .
$$

Proof. (a) Note that the mappings

$$
I: \mathcal{H}\left(E_{1}, E_{0}\right) \rightarrow \mathcal{L}\left(\mathbb{E}_{1}(J), \mathbb{E}_{0}(J) \times E_{\mu}\right), \quad A \mapsto\left(\frac{d}{d t}+A, \gamma\right),
$$

Inv : $\operatorname{Isom}\left(\mathbb{E}_{1}(J), \mathbb{E}_{0}(J) \times E_{\mu}\right) \rightarrow \mathcal{L}\left(\mathbb{E}_{0}(J) \times E_{\mu}, \mathbb{E}_{1}(J)\right)$,

$$
\left(\frac{d}{d t}+A, \gamma\right) \mapsto\left(\frac{d}{d t}+A, \gamma\right)^{-1}
$$

are real analytic. Moreover, $\mathcal{M}_{\mu}\left(E_{1}, E_{0}\right)=I^{-1}\left(\operatorname{Isom}\left(\mathbb{E}_{1}(J), \mathbb{E}_{0}(J) \times E_{\mu}\right)\right)$. The first assertion follows from the fact that $\operatorname{Isom}\left(\mathbb{E}_{1}(J), \mathbb{E}_{0}(J) \times E_{\mu}\right)$ is open in $\mathcal{L}\left(\mathbb{E}_{1}(J), \mathbb{E}_{0}(J) \times E_{\mu}\right)$.

(b) It follows from part (a) that the set $\left\{\left(\frac{d}{d t}+A, \gamma\right)^{-1} ; A \in \mathcal{A}\right\}$ is compact, and thus bounded, in $\mathcal{L}\left(\mathbb{E}_{0}(J) \times E_{\mu}, \mathbb{E}_{1}(J)\right)$.

LEMMA 2.6. $\mathcal{M}_{\mu}\left(E_{1}, E_{0}\right) \subset \mathcal{M}_{\sigma}\left(E_{1}, E_{0}\right)$ for $0<\mu<\sigma \leq 1$.

Proof. (i) We first consider the case $\sigma<1$. Suppose that $A \in \mathcal{M}_{\mu}\left(E_{1}, E_{0}\right)$ and let $f \in B U C_{1-\sigma}\left(J, E_{0}\right)$ be given. Then

$$
\begin{aligned}
& \int_{0}^{t} e^{-(t-\tau) A} f(\tau) d \tau=t^{\sigma-\mu} \int_{0}^{t} e^{-(t-\tau) A} \frac{1}{\tau^{\sigma-\mu}} f(\tau) d \tau \\
& +\int_{0}^{t} e^{-(t-\tau) A} \frac{\left(\tau^{\sigma-\mu}-t^{\sigma-\mu}\right)}{\tau^{\sigma-\mu}} f(\tau) d \tau=: g_{1}(t)+g_{2}(t) .
\end{aligned}
$$

It is clear that the function $\left[\tau \mapsto \tau^{\mu-\sigma} f(\tau)\right]$ belongs to $B U C_{1-\mu}\left(J, E_{0}\right)$ and we conclude from Remark 2.4(c) that the first integral belongs to $B U C_{1-\mu}\left(J, E_{1}\right)$. This implies that $g_{1} \in B U C_{1-\sigma}\left(J, E_{1}\right)$. For the second integral we have

$$
\begin{aligned}
& \int_{0}^{t}\left\|e^{-(t-\tau) A} \frac{\left(\tau^{\sigma-\mu}-t^{\sigma-\mu}\right)}{\tau^{\sigma-\mu}} f(\tau)\right\|_{E_{1}} d \tau \\
& \quad \leq c \int_{0}^{t} \frac{d \tau}{(t-\tau)^{1-(\sigma-\mu)} \tau^{1-\mu}} \sup _{0<\tau \leq t} \tau^{1-\sigma}\|f(\tau)\|_{E_{0}} \\
& \quad=c \frac{1}{t^{1-\sigma}} \int_{0}^{1} \frac{d s}{(1-s)^{1-(\sigma-\mu)} s^{1-\mu}} \sup _{0<\tau \leq t} \tau^{1-\sigma}\|f(\tau)\|_{E_{0}}
\end{aligned}
$$


and we can conclude that $g_{2} \in B U C_{1-\sigma}\left(J, E_{1}\right)$. Therefore, $K_{A} f \in B U C_{1-\sigma}\left(J, E_{1}\right)$ and the assertion follows from (c).

(ii) We now consider the case $\sigma=1$. Let $f \in B U C_{0}\left(J, E_{0}\right)=B U C\left(J, E_{0}\right)$ be given. We can assume that $f(0)=0$. Otherwise we write $f=f-f(0)+f(0)$. Since $A\left(K_{A} f(0)\right)=\left(I-e^{i t-t A}\right) f(0) \in B U C\left(J, E_{0}\right)$ we obtain $K_{A} f(0) \in B U C\left(J, E_{1}\right)$. The condition $f(0)=0$ implies that $\left[\tau \mapsto \tau^{\mu-1} f(\tau)\right]$ belongs to $B U C_{1-\mu}\left(J, E_{0}\right)$ and we can now repeat the proof given in (i).

LEMMA 2.7. (a) Suppose that $\left(\frac{d}{d t}+A, \gamma\right) \in \operatorname{Isom}\left(\mathbb{E}_{1}(J), \mathbb{E}_{0}(J) \times E_{\mu}\right)$ for $J=$ $[0, T]$. Then $\left(\frac{d}{d t}+A, \gamma\right) \in \operatorname{Isom}\left(\mathbb{E}_{1}\left(J_{\tau}\right), \mathbb{E}_{0}\left(J_{\tau}\right) \times E_{\mu}\right)$ for any subinterval $J_{\tau}=$ $[0, \tau] \subset J$ and

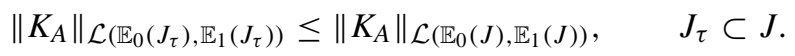

(b) Suppose that $\left(\frac{d}{d t}+A, \gamma\right) \in \operatorname{Isom}\left(\mathbb{E}_{1}(J), \mathbb{E}_{0}(J) \times E_{\mu}\right)$ for $J=[0, T]$. Then $\left(\frac{d}{d t}+\right.$ $A, \gamma) \in \operatorname{Isom}\left(\mathbb{E}_{1}(I), \mathbb{E}_{0}(I) \times E_{\mu}\right)$ for any finite interval $I=\left[0, T_{1}\right]$.

(c) Let $E_{\gamma}:=\left(E_{0}, E_{1}\right)_{\gamma}, \gamma \in(0,1)$, be an interpolation space, where $(\cdot, \cdot)_{\gamma}$ is an arbitrary interpolation functor of exponent $\gamma$. Suppose that $A \in \mathcal{M}_{\mu}\left(E_{1}, E_{0}\right)$ and that $B \in \mathcal{L}\left(E_{\gamma}, E_{0}\right)$ for some $0 \leq \gamma<1$. Then $A+B \in \mathcal{M}_{\mu}\left(E_{1}, E_{0}\right)$.

Proof. These results are contained in [4, Lemmas 2.3-2.5]. For the reader's convenience we include a proof.

(a) Let $f \in B U C_{1-\mu}\left(J_{\tau}\right)$ be given. Then we set

$$
(\mathcal{E} f)(t):= \begin{cases}f(t) & \text { if } 0<t \leq \tau, \\ \left(\frac{\tau}{t}\right)^{1-\mu} f(\tau) & \text { if } \tau \leq t \leq T .\end{cases}
$$

It easy to see that $\mathcal{E} \in \mathcal{L}\left(B U C_{1-\mu}\left(J_{\tau}, E_{0}\right), B U C_{1-\mu}\left(J, E_{0}\right)\right)$ with $\|\mathcal{E}\|=1$. The assertions follow from Remark 2.4(c) and the fact that $K_{A, \tau}=\mathcal{R} \circ K_{A, T} \circ \mathcal{E}$, where $\mathcal{R}$ denotes the restriction operator $\mathcal{R} u:=\left.u\right|_{J_{\tau}}$.

(b) Let $I=\left[0, T_{1}\right]$ be an arbitrary interval. Due to part (a) we may assume that $J \subset I$. Then there exists a number $n \in \mathbb{N}^{*}$ such that $I \subset[0, n T]$. Let $(f, x) \in \mathbb{E}_{0}([0, n T]) \times$ $E_{\mu}$ be given and let

$$
\begin{aligned}
& f_{0}:=\left.f\right|_{J}, \quad v_{0}:=\left(\frac{d}{d t}+A, \gamma\right)^{-1}\left(f_{0}, x\right) \\
& f_{j}:=\left.f(\cdot+j T)\right|_{J}, \quad v_{j}:=\left(\frac{d}{d t}+A, \gamma\right)^{-1}\left(f_{j}, v_{j-1}(T)\right)
\end{aligned}
$$

for $j=1, \ldots, n-1$. It follows from our assumptions and from Lemma 2.6 that $v_{0} \in B U C_{1-\mu}^{1}\left(J, E_{0}\right) \cap B U C_{1-\mu}\left(J, E_{1}\right), \quad v_{j} \in B U C^{1}\left(J, E_{0}\right) \cap B U C\left(J, E_{1}\right)$ 
for $j=1, \ldots, n-1$. It is now easy to verify that the function $v$ defined by $v(t):=v_{j}(t-j T) \quad$ if $j T \leq t \leq(j+1) T, \quad j \in\{0, \ldots, n-1\}, \quad t \in[0, n T]$, belongs to $\mathbb{E}_{1}([0, n T])$ and satisfies $\left(\frac{d}{d t}+A, \gamma\right) v=(f, x)$. The assertion follows from Remark 2.4(a), the open mapping theorem, and part (a) of the proof.

(c) (i) A well-known perturbation result for generators of analytic semigroups yields $A+B \in \mathcal{H}\left(E_{1}, E_{0}\right)$. According to Lemma 2.7(b) is suffices to show that $\left(\frac{d}{d t}+A+B, \gamma\right) \in \operatorname{Isom}\left(\mathbb{E}_{1}\left(J_{1}\right), \mathbb{E}_{0}\left(J_{1}\right) \times E_{\mu}\right), \quad J_{1}=\left[0, t_{1}\right]$

for some number $t_{1} \in \dot{J}$.

(ii) Let $f \in \mathbb{E}_{0}\left(J_{1}\right)$ be given. Then there exists a constant $M=M(J) \geq 1$ such that

$$
\begin{gathered}
t^{1-\mu}\left\|B\left(K_{A} f\right)(t)\right\|_{E_{0}} \leq\|B\|_{\mathcal{L}\left(E_{\gamma}, E_{0}\right)} t^{1-\mu}\left\|\left(K_{A} f\right)(t)\right\|_{E_{\gamma}} \\
\quad \leq M t^{1-\mu} \int_{0}^{t} \frac{1}{(t-\tau)^{\gamma} \tau^{1-\mu}} d \tau \sup _{0<\tau \leq t_{1}} \tau^{1-\mu}\|f(\tau)\|_{E_{0}} \\
\quad=M t^{1-\gamma} \int_{0}^{1} \frac{1}{(1-\tau)^{\gamma} \tau^{1-\mu}} d \tau \sup _{0<\tau \leq t_{1}} \tau^{1-\mu}\|f(\tau)\|_{E_{0}}
\end{gathered}
$$

for any $t \in J_{1}$ and any $t_{1} \in \dot{J}$. Hence $\left[\mathrm{id}+B K_{A}\right] \in \operatorname{Isom}\left(\mathbb{E}_{0}\left(J_{1}\right)\right)$, provided $t_{1}$ is chosen small enough.

(iii) Given $(f, x) \in \mathbb{E}_{0}\left(J_{1}\right) \times E_{\mu}$ we set $u(t):=e^{-t(A+B)} x+K_{A}\left[i d+B K_{A}\right]^{-1} f$ for $t \in J_{1}$. It follows from Remark 2.1(c) and the Proof of Remark 2.4(c) that $u \in \mathbb{E}_{1}\left(J_{1}\right)$. Moreover,

$$
\left(\frac{d}{d t}+A+B, \gamma\right) u=(f, x)
$$

and we conclude that $\left(\frac{d}{d t}+A+B, \gamma\right) \in \operatorname{Isom}\left(\mathbb{E}_{1}\left(J_{1}\right), \mathbb{E}_{0}\left(J_{1}\right) \times E_{\mu}\right)$.

LEMMA 2.8. (a) Suppose that $[s \mapsto A(s)] \in B U C\left(J, \mathcal{L}\left(E_{1}, E_{0}\right)\right)$ and that

$$
\left(\frac{d}{d t}+A(s), \gamma\right) \in \operatorname{Isom}\left(\mathbb{E}_{1}(J), \mathbb{E}_{0}(J) \times E_{\mu}\right), \quad s \in J=[0, T] .
$$

\section{Then}

$$
\left(\frac{d}{d t}+A(\cdot), \gamma\right) \in \operatorname{Isom}\left(\mathbb{E}_{1}(J), \mathbb{E}_{0}(J) \times E_{\mu}\right) .
$$

(b) Suppose that the family $\{A(s) ; s \in J\}$ satisfies the assumptions of (a) and that $R \in B U C_{1-\mu}\left(J, \mathcal{L}\left(E_{\mu}, E_{0}\right)\right)$ with $\mu \in(0,1)$. Then

$$
\left(\frac{d}{d t}+A(\cdot)+R(\cdot), \gamma\right) \in \operatorname{Isom}\left(\mathbb{E}_{1}(J), \mathbb{E}_{0}(J) \times E_{\mu}\right) \text {. }
$$


Proof. (a) We refer to [3, Theorem III.2.6.1 and Remark III.3.4.2(c)].

(b) (i) We can write

$$
\begin{gathered}
\left(\frac{d}{d t}+A(\cdot)+R(\cdot), \gamma\right)=[(\mathrm{id}, \mathrm{id})+(R(\cdot)+A(\cdot)-A(0), 0) \\
\left.\left(\frac{d}{d t}+A_{0}, \gamma\right)^{-1}\right]\left(\frac{d}{d t}+A_{0}, \gamma\right)
\end{gathered}
$$

where $A_{0}:=A(0)$ and where (id, id) $:=\left(\operatorname{id}_{\mathbb{E}_{0}(J)}, \operatorname{id}_{E_{\mu}}\right)$. Let $(f, x) \in \mathbb{E}_{1}(J) \times$ $E_{\mu}$ be given. There exists a constant $M=M(J) \geq 1$ such that

$$
\begin{aligned}
& t^{1-\mu}\left\|(A(t)-A(0))\left(\frac{d}{d t}+A_{0}, \gamma\right)^{-1}(f, x)\right\|_{E_{0}} \\
& \quad \leq M \sup _{\tau \in\left[0, t_{1}\right]}\|A(\tau)-A(0)\|_{\mathcal{L}\left(E_{1}, E_{0}\right)}\|(f, x)\|_{\mathbb{E}_{0}\left(\left[0, t_{1}\right]\right) \times E_{\mu}}
\end{aligned}
$$

and

$$
\begin{aligned}
& t^{1-\mu}\left\|R(t)\left(\frac{d}{d t}+A_{0}, \gamma\right)^{-1}(f, x)\right\|_{E_{0}} \\
& \quad \leq t^{1-\mu}\|R(t)\|_{\mathcal{L}\left(E_{\mu}, E_{0}\right)}\left(\left\|\left(K_{A_{0}} f\right)(t)\right\|_{E_{\mu}}+\left\|e^{-t A_{0}} x\right\|_{E_{\mu}}\right) \\
& \quad \leq M \sup _{\tau \in\left[0, t_{1}\right]} \tau^{1-\mu}\|R(\tau)\|_{\mathcal{L}\left(E_{\mu}, E_{0}\right)}\|(f, x)\|_{\mathbb{E}_{0}\left(\left[0, t_{1}\right]\right) \times E_{\mu}}
\end{aligned}
$$

for any $t_{1} \in \dot{J}$ and $t \in\left(0, t_{1}\right]$. The estimates imply that

$$
\begin{aligned}
& \left\|(R(\cdot)+A(\cdot)-A(0), 0)\left(\frac{d}{d t}+A_{0}, \gamma\right)^{-1}\right\|_{\mathcal{L}\left(\mathbb{E}_{0}\left(J_{1}\right) \times E_{\mu}\right)} \\
& \quad \leq 1 / 2, \quad J_{1}:=\left[0, t_{1}\right],
\end{aligned}
$$

provided $t_{1}$ is sufficiently small and we conclude that $\left(\frac{d}{d t}+A(\cdot)+R(\cdot), \gamma\right) \in \operatorname{Isom}\left(\mathbb{E}_{1}\left(J_{1}\right), \mathbb{E}_{0}\left(J_{1}\right) \times E_{\mu}\right)$.

(ii) It follows from our assumptions and from Lemma 2.6 and Lemma 2.7(c) that the family $\left\{\left(A\left(t_{1}+s\right)+R\left(t_{1}+s\right)\right) ; s \in J_{2}\right\}, J_{2}:=\left[0, T-t_{1}\right]$, satisfies the assumptions of part (a) with $\mu=1$. We conclude that

$$
\begin{aligned}
& \left(\frac{d}{d t}+A\left(t_{1}+\cdot\right)+R\left(t_{1}+\cdot\right), \gamma\right) \in \operatorname{Isom}\left(C^{1}\left(J_{2}, E_{0}\right)\right. \\
& \left.\cap C\left(J_{2}, E_{0}\right), C\left(J_{2}, E_{0}\right) \times E_{1}\right) .
\end{aligned}
$$

(iii) The assertion follows now from (i)-(ii). 
REMARK 2.9. Lemma 2.2(a) was proved in [7], see also [12, Appendix]. In these publications, the notation $D_{A}(\theta)$ is usually used instead of $E_{\theta}$. Lemma 2.2(b) was first established in [15], see also the remark following the proof of [3, Theorem III.2.3.3]. The fact that $\mathcal{M}_{\mu}\left(E_{1}, E_{0}\right)$ is open in $\mathcal{H}\left(E_{1}, E_{0}\right)$ was shown in [4, Lemma 2.1]. Lemma 2.6 was proved in [4] for the case $\sigma=1$.

\section{Local existence and uniqueness}

In this section we study the existence of solutions to the quasilinear parabolic Problem (1.1). To do so we will first introduce the notion of a solution. We assume that

$$
(A, f): V \rightarrow \mathcal{H}\left(E_{1}, E_{0}\right) \times E_{0}
$$

where $V$ is a nonempty subset of $E_{0}$. Let $x \in V$ be given and let $J \subset \mathbb{R}^{+}:=[0, \infty)$ be an interval which contains 0 . By a solution $u$ of (1.1) on $J$ we mean a function

$$
u \in C^{1}\left(\dot{J}, E_{0}\right) \cap C\left(\dot{J}, E_{1}\right) \cap C(J, V)
$$

which satisfies

$$
\left\{\begin{array}{l}
\dot{u}(t)+A(u(t)) u(t)=f(u(t)), \quad t \in \dot{J}, \\
u(0)=x,
\end{array}\right.
$$

where $\dot{J}=J \backslash\{0\}$. We are ready to prove the following fundamental local existence, uniqueness and continuity theorem for quasilinear parabolic evolution equations.

THEOREM 3.1. Let $\alpha \in(0,1)$ be fixed and let $E_{\alpha}:=\left(E_{0}, E_{1}\right)_{\alpha}$ be a continuous interpolation space. Assume that $V_{\alpha} \subset E_{\alpha}$ is open and that

$$
(A, f) \in C^{1-}\left(V_{\alpha}, \mathcal{M}_{\alpha}\left(E_{1}, E_{0}\right) \times E_{0}\right) .
$$

(a) For every $x_{0}$ in $V_{\alpha}$ there exist positive constants $\tau=\tau\left(x_{0}\right), \varepsilon=\varepsilon\left(x_{0}\right)$ and $c=c\left(x_{0}\right)$ such that the quasilinear evolution equation (1.1) has a unique solution

$u(\cdot, x) \in B U C_{1-\alpha}^{1}\left([0, \tau], E_{0}\right) \cap B U C_{1-\alpha}\left([0, \tau], E_{1}\right)$

on $[0, \tau]$ for any initial value $x \in \overline{\mathbb{B}}_{E_{\alpha}}\left(x_{0}, \varepsilon\right)$. Moreover,

$u(\cdot, x) \in B U C\left([0, \tau], V_{\alpha}\right) \cap B U C^{\alpha-\beta}\left([0, \tau], E_{\beta}\right)$

for any $\beta \in[0, \alpha)$, where $E_{\beta}:=\left(E_{0}, E_{1}\right)_{\beta}$, and

$$
\|u(\cdot, x)-u(\cdot, y)\|_{C\left([0, \tau], E_{\alpha}\right)} \leq c\|x-y\|_{E_{\alpha}}, \quad x, y \in \overline{\mathbb{B}}_{E_{\alpha}}\left(x_{0}, \varepsilon\right) .
$$


(b) Let $S \subset V_{\alpha}$ be compact. Then there exist bounded closed neighborhoods $U$ and $W$ of $S$ and constants $\tau, c>0$ such that

- $S \subset U \subset \stackrel{\circ}{W} \subset W \subset V_{\alpha}$

- The quasilinear Problem (1.1) has for each $x \in U$ a unique solution $u(\cdot, x) \in B U C_{1-\alpha}^{1}\left([0, \tau], E_{0}\right) \cap B U C_{1-\alpha}\left([0, \tau], E_{1}\right) \cap B U C\left([0, \tau], V_{\alpha}\right)$ on $[0, \tau]$ and $\|u(\cdot, x)-u(\cdot, y)\|_{C\left([0, \tau], E_{\alpha}\right)} \leq c\|x-y\|, \quad x, y \in U$.

Proof. (a) In the following we set

$$
\begin{aligned}
& \mathbb{E}_{0}(J):=B U C_{1-\alpha}\left(J, E_{0}\right), \\
& \mathbb{E}_{1}(J):=B U C_{1-\alpha}^{1}\left(J, E_{0}\right) \cap B U C_{1-\alpha}\left(J, E_{1}\right)
\end{aligned}
$$

where the meaning of the interval $J$ will be evident from the context. It is clear that Problem (1.1) is equivalent to the evolution equation

$\left\{\begin{array}{l}\dot{u}+A u=B(u) u+f(u), \\ u(0)=x,\end{array}\right.$

where $A:=A\left(x_{0}\right)$ and $B(z):=A\left(x_{0}\right)-A(z)$ for $z \in V_{\alpha}$. We conclude that $B \in C^{1-}\left(V_{\alpha}, \mathcal{L}\left(E_{1}, E_{0}\right)\right)$ and that $B\left(x_{0}\right)=0$. In the following we assume that $E_{\alpha}$ is equipped with the (equivalent) norm

$\|\cdot\|_{E_{\alpha}}:=\sup _{s>0} s^{1-\alpha}\left\|(\omega+A) e^{-s(\omega+A)} \cdot\right\|_{E_{0}}$

where $\omega$ is a fixed number such that type $(-(\omega+A))<0$. Let $T>0$ be fixed and let $J:=[0, T]$. It follows from Lemma 2.2(c) that there exists a constant $M_{1} \geq 1$ such that

$\|u\|_{C\left(J_{\tau}, E_{\alpha}\right)} \leq M_{1}\|u\|_{\mathbb{E}_{1}\left(J_{\tau}\right)}, \quad u \in \mathbb{E}_{1}\left(J_{\tau}\right), \quad u(0)=0, \quad J_{\tau}=[0, \tau] \subset J$.

Moreover, we obtain

$\left\|e^{-t A} z\right\|_{\mathbb{E}_{1}\left(J_{\tau}\right)} \leq c(\omega) e^{\omega T} \sup _{s>0} s^{1-\alpha}\left\|(\omega+A) e^{-s(\omega+A)} z\right\|_{E_{0}} \leq M_{2}\|z\|_{E_{\alpha}}$

for $z \in E_{\alpha}$ and $J_{\tau} \subset J$. Let $\left\|K_{A}\right\|:=\left\|K_{A}\right\|_{\mathcal{L}\left(\mathbb{E}_{0}(J), \mathbb{E}_{1}(J)\right)}$. Then there exist positive constants $\rho_{0}, b$, and $L \geq 1$ such that $\mathbb{B}_{E_{\alpha}}\left(x_{0}, 2 \rho_{0}\right) \subset V_{\alpha}$ and such that

$$
\begin{gathered}
\|B(z)\|_{\mathcal{L}\left(E_{1}, E_{0}\right)} \leq \frac{1}{4\left\|K_{A}\right\| M_{1}}, \quad\|f(z)\| \leq b, \quad z \in \overline{\mathbb{B}}_{E_{\alpha}}\left(x_{0}, \rho_{0}\right), \\
\left\|(B, f)\left(z_{1}\right)-(B, f)\left(z_{2}\right)\right\|_{\mathcal{L}\left(E_{1}, E_{0}\right) \times E_{0}} \leq L\left\|z_{1}-z_{2}\right\|_{E_{\alpha}},
\end{gathered}
$$


where $z_{1}, z_{2} \in \overline{\mathbb{B}}_{E_{\alpha}}\left(x_{0}, \rho_{0}\right)$. Let $\varepsilon_{0}:=\min \left(\rho_{0},\left(4\left\|K_{A}\right\| M_{1} L\right)^{-1}\right)$. Then we find a number $T_{1} \in J$ such that

$$
\begin{gathered}
\left\|e^{-t A} x_{0}-x_{0}\right\|_{E_{\alpha}} \leq \varepsilon_{0} / 2, \quad t \in J_{1}:=\left[0, T_{1}\right], \\
\left\|e^{-t A} x_{0}\right\|_{\mathbb{E}_{1}\left(J_{1}\right)} \leq \varepsilon_{0} / 2 .
\end{gathered}
$$

The first inequality in (3.9) follows from the strong continuity of the semigroup $\left\{e^{-t A} ; t \geq 0\right\}$ on $E_{\alpha}$, whereas the second one is a consequence of Remark 2.1(c). Let $\tau \leq T_{1}$ be given and set $J_{\tau}=[0, \tau]$. For $x \in \overline{\mathbb{B}}_{E_{\alpha}}(0, \varepsilon)$ with $2 M_{2} \varepsilon \leq \varepsilon_{0}$ we set $W_{x}\left(J_{\tau}\right):=\left\{v \in \mathbb{E}_{1}\left(J_{\tau}\right) ; v(0)=x,\left\|v-x_{0}\right\|_{C\left(J_{\tau}, E_{\alpha}\right)} \leq \varepsilon_{0}\right\} \cap \overline{\mathbb{B}}_{\mathbb{E}_{1}\left(J_{\tau}\right)}\left(0, \varepsilon_{0}\right)$

and equip this set with the topology of $\mathbb{E}_{1}\left(J_{\tau}\right)$. It follows from Lemma 2.2(b) that $W_{x}\left(J_{\tau}\right)$ is a closed subset of $\mathbb{E}_{1}\left(J_{\tau}\right)$ and thus is a complete metric space. (3.7) and (3.9) yield $\left[t \mapsto e^{-t A} x\right] \in W_{x}\left(J_{\tau}\right)$, showing that $W_{x}\left(J_{\tau}\right)$ is nonempty. Let $v \in W_{x}\left(J_{\tau}\right)$ be given. Then we obtain from (3.8)

$$
\begin{aligned}
& t^{1-\alpha}\|B(v(t)) v(t)+f(v(t))\|_{E_{0}} \\
& \left.\quad \leq\|B(v(t))\|_{\mathcal{L}\left(E_{1}, E_{0}\right)}\right)^{1-\alpha}\|v(t)\|_{E_{1}}+t^{1-\alpha}\|f(v(t))\|_{E_{0}} \\
& \quad \leq \frac{1}{4\left\|K_{A}\right\| M_{1}}\|v\|_{\mathbb{E}_{1}\left(J_{\tau}\right)}+\tau^{1-\alpha} b \\
& \quad \leq \frac{\varepsilon_{0}}{4\left\|K_{A}\right\| M_{1}}+\tau^{1-\alpha} b, \quad t \in J_{\tau} .
\end{aligned}
$$

It follows from Lemma 2.2(b), the mapping properties of $(B, f)$, and the estimates above that $B(v) v+f(v) \in \mathbb{E}_{0}\left(J_{\tau}\right)$ for any $v \in W_{x}\left(J_{\tau}\right)$. (2.9) and Remark 2.4(b) imply that the mapping

$$
G_{x}: W_{x}\left(J_{\tau}\right) \rightarrow \mathbb{E}_{1}\left(J_{\tau}\right), \quad G_{x}(v):=e^{-t A} x+K_{A}(B(v) v+f(v))
$$

is well defined for any $x \in \overline{\mathbb{B}}_{E_{\alpha}}(0, \varepsilon)$.

(i) It follows from (3.6), (3.9)-(3.10), and from the strong continuity of the semigroup $\left\{e^{-t A} ; t \geq 0\right\}$ on $E_{\alpha}$ that

$$
\begin{aligned}
&\left\|G_{x}(v)-x_{0}\right\|_{C\left(J_{\tau}, E_{\alpha}\right)} \\
& \leq\left\|e^{-t A}\left(x-x_{0}\right)\right\|_{C\left(J_{\tau}, E_{\alpha}\right)}+\left\|e^{-t A} x_{0}-x_{0}\right\|_{C\left(J_{\tau}, E_{\alpha}\right)} \\
&+\left\|K_{A}(B(v) v+f(v))\right\|_{C\left(J_{\tau}, E_{\alpha}\right)} \\
& \leq c\left\|x-x_{0}\right\|_{E_{\alpha}}+\frac{\varepsilon_{0}}{2}+M_{1}\left\|K_{A}\right\|\left(\frac{\varepsilon_{0}}{4\left\|K_{A}\right\| M_{1}}+\tau^{1-\alpha} b\right) \leq \varepsilon_{0},
\end{aligned}
$$

provided that $\left\|x-x_{0}\right\|_{E_{\alpha}} \leq \varepsilon$ for a sufficiently small number $\varepsilon$ and provided that $\tau$ is small enough. We can always make $\tau$ smaller since the relevant constants 
and $\left\|K_{A}\right\|_{\mathcal{L}\left(\mathbb{E}_{0}\left(J_{\tau}\right), \mathbb{E}_{1}\left(J_{\tau}\right)\right)}$ are independent of $J_{\tau} \subset J$, see Lemma 2.7(a). Additionally, we also obtain

$$
\begin{aligned}
& \left\|G_{x}(v)\right\|_{\mathbb{E}_{1}\left(J_{\tau}\right)} \leq\left\|e^{-t A}\left(x-x_{0}\right)\right\|_{\mathbb{E}_{1}\left(J_{\tau}\right)}+\left\|e^{-t A} x_{0}\right\|_{\mathbb{E}_{1}\left(J_{\tau}\right)} \\
& +\left\|K_{A}(B(v)+f(v))\right\|_{\mathbb{E}_{1}\left(J_{\tau}\right)} \\
& \leq M_{2}\left\|x-x_{0}\right\|_{E_{\alpha}}+\frac{\varepsilon_{0}}{2}+\left\|K_{A}\right\| \\
& \left(\frac{\varepsilon_{0}}{4\left\|K_{A}\right\| M_{1}}+\tau^{1-\alpha} b\right) \leq \varepsilon_{0}
\end{aligned}
$$

if $\varepsilon$ and $\tau$ are small enough. Lastly, observe that $G_{x}(v)(0)=x$. We have shown that $G_{x}\left(W_{x}\left(J_{\tau}\right)\right) \subset W_{x}\left(J_{\tau}\right)$ for all $x \in \overline{\mathbb{B}}_{E_{\alpha}}\left(x_{0}, \varepsilon\right)$, provided that $\varepsilon$ and $\tau$ are sufficiently small.

(ii) Let $x_{1}, x_{2} \in \overline{\mathbb{B}}_{E_{\alpha}}\left(x_{0}, \varepsilon\right)$ be given and pick $v_{1} \in W_{x_{1}}\left(J_{\tau}\right)$ and $v_{2} \in W_{x_{2}}\left(J_{\tau}\right)$. It follows from (3.7) that

$\left\|e^{-t A}\left(x_{1}-x_{2}\right)\right\|_{\mathbb{E}_{1}\left(J_{\tau}\right)} \leq M_{2}\left\|x_{1}-x_{2}\right\|_{E_{\alpha}}$.

Moreover, we obtain from (3.6) that

$\left\|\left(v_{1}-v_{2}\right)-e^{-t A}\left(x_{1}-x_{2}\right)\right\|_{C\left(J_{\tau}, E_{\alpha}\right)} \leq M_{1} \|$

$$
\left(v_{1}-v_{2}\right)-e^{-t A}\left(x_{1}-x_{2}\right) \|_{\mathbb{E}_{1}\left(J_{\tau}\right)} \text {. }
$$

This estimate together with (3.11) immediately yields

$$
\begin{aligned}
& \left\|v_{1}-v_{2}\right\|_{C\left(J_{\tau}, E_{\alpha}\right)} \leq M_{1}\left\|v_{1}-v_{2}\right\|_{\mathbb{E}_{1}\left(J_{\tau}\right)} \\
& \quad+M_{2}\left(1+M_{1}\right)\left\|x_{1}-x_{2}\right\|_{E_{\alpha}} .
\end{aligned}
$$

Next observe that

$$
\begin{aligned}
\left\|B\left(v_{1}\right)\left(v_{1}-v_{2}\right)\right\|_{\mathbb{E}_{0}\left(J_{\tau}\right)} & \leq \frac{1}{4\left\|K_{A}\right\| M_{1}}\left\|v_{1}-v_{2}\right\|_{\mathbb{E}_{1}\left(J_{\tau}\right)}, \\
\left\|\left(B\left(v_{1}\right)-B\left(v_{2}\right)\right) v_{2}\right\|_{\mathbb{E}_{0}\left(J_{\tau}\right)} & \leq L\left\|v_{1}-v_{2}\right\|_{C\left(J, E_{\alpha}\right)}\left\|v_{2}\right\|_{\mathbb{E}_{1}\left(J_{\tau}\right)} \\
& \leq \varepsilon_{0} L\left\|v_{1}-v_{2}\right\|_{C\left(J_{\tau}, E_{\alpha}\right)} \\
\left\|f\left(v_{1}\right)-f\left(v_{2}\right)\right\|_{\mathbb{E}_{0}\left(J_{\tau}\right)} & \leq L \tau^{1-\alpha}\left\|v_{1}-v_{2}\right\|_{C\left(J_{\tau}, E_{\alpha}\right)} .
\end{aligned}
$$

If follows from the definition of $\varepsilon_{0}$ and from (3.11)-(3.13) that there exists a constant $c_{2}>0$ such that

$$
\begin{gathered}
\left\|G_{x_{1}}\left(v_{1}\right)-G_{x_{2}}\left(v_{2}\right)\right\|_{\mathbb{E}_{1}\left(J_{\tau}\right)} \leq c_{2}\left\|x_{1}-x_{2}\right\|_{E_{\alpha}} \\
+\left(\frac{1}{2}+\left\|K_{A}\right\| M_{1} L \tau^{1-\alpha}\right)\left\|v_{1}-v_{2}\right\|_{\mathbb{E}_{1}\left(J_{\tau}\right)} \\
\quad \leq c_{2}\left\|x_{1}-x_{2}\right\|_{E_{\alpha}}+\frac{3}{4}\left\|v_{1}-v_{2}\right\|_{\mathbb{E}_{1}\left(J_{\tau}\right)}
\end{gathered}
$$

provided that $\tau$ is chosen small enough. 
(iii) As a particular case we obtain from (3.14) that

$$
\begin{gathered}
\left\|G_{x}\left(v_{1}\right)-G_{x}\left(v_{2}\right)\right\|_{\mathbb{E}_{1}\left(J_{\tau}\right)} \leq \frac{3}{4}\left\|v_{1}-v_{2}\right\|_{\mathbb{E}_{1}\left(J_{\tau}\right)}, \\
x \in \overline{\mathbb{B}}_{E_{\alpha}}\left(x_{0}, \varepsilon\right), \quad v_{1}, v_{2} \in W_{x}\left(J_{\tau}\right) .
\end{gathered}
$$

(iv) It follows from (i)-(iii) and Banach's fixed point theorem that the mapping $G_{x}$ has a unique fixed point

$u(\cdot, x) \in W_{x}\left(J_{\tau}\right) \subset B U C_{1-\alpha}^{1}\left(J_{\tau}, E_{0}\right) \cap B U C_{1-\alpha}\left(J_{\tau}, E_{1}\right)$

for each $x \in \overline{\mathbb{B}}_{E_{\alpha}}\left(x_{0}, \varepsilon\right)$. (3.2) and the additional regularity assertions follow now from (3.15) and Lemma 2.2(d).

(v) We infer from (3.14) that

$$
\begin{aligned}
& \|u(\cdot, x)-u(\cdot, y)\|_{\mathbb{E}_{1}\left(J_{\tau}\right)} \leq 4 c_{2}\|x-y\|_{E_{\alpha}}, \quad x, y \in \overline{\mathbb{B}}_{E_{\alpha}}\left(x_{0}, \varepsilon\right) \\
& \text { and (3.4) follows from Lemma 2.2(d). }
\end{aligned}
$$

(vi) Suppose $u_{1}$ and $u_{2}$ are two solutions of (1.1) which satisfy (3.2). Let

$\tau_{1}:=\sup \left\{t \in[0, \tau] ; u_{1}(s)=u_{2}(s) \quad \forall 0 \leq s<t\right\}$.

Since $u_{1}$ and $u_{2}$ satisfy (3.2) we conclude that both belong to the set

$\left\{v \in \mathbb{E}_{1}\left(J_{\tau^{*}}\right) ; v(0)=x,\|v-x\|_{C\left(J_{\tau^{*}}, E_{\alpha}\right)} \leq \varepsilon_{0}\right\} \cap \overline{\mathbb{B}}_{\mathbb{E}_{1}\left(J_{\tau^{*}}\right)}\left(0, \varepsilon_{0}\right)$

provided $\tau^{*}$ is sufficiently small. Banach's fixed point theorem provides a unique solution in this set and we conclude that $\tau_{1}>0$. Assume that $\tau_{1}<\tau$. It is clear that $u_{1}\left(\tau_{1}\right)=u_{2}\left(\tau_{1}\right)=: y$. Let $v_{j}(t):=u_{j}\left(t+\tau_{1}\right), j=1,2$, with $t \in J_{2}:=$ $\left[0, \tau_{2}\right]$ for some $\tau_{2} \in\left(0, \tau-\tau_{1}\right]$. Then $v_{1}, v_{2} \in C^{1}\left(J_{2}, E_{0}\right) \cap C\left(J_{2}, E_{1}\right)$, and $v_{1}, v_{2}$ solve

$\dot{u}+A(u) u=f(u), \quad u(0)=y$.

If $\tau_{2}$ is small enough, then $v_{1}$ and $v_{2}$ belong to the set

$\left\{v \in \mathbb{E}_{1}\left(J_{2}\right) ; v(0)=y,\|v-y\|_{C\left(J_{2}, E_{\alpha}\right)} \leq \varepsilon_{0}\right\} \cap \overline{\mathbb{B}}_{\mathbb{E}_{1}\left(J_{2}\right)}\left(0, \varepsilon_{0}\right)$

and we conclude once again that $v_{1}=v_{2}$. Therefore, $u_{1}$ and $u_{2}$ coincide on the interval $\left[0, \tau_{1}+\tau_{2}\right]$, thus contradicting the definition of $\tau_{1}$.

(b) Part (b) follows from part (a) by a compactness argument.

REMARK 3.2. (a) Theorem 3.1 shows that solutions of (1.1) starting out in $E_{\alpha}$ immediately regularize and are in $E_{1}$ for any positive time $t>0$. This is an important feature of quasilinear parabolic evolution equations which has far reaching consequences for questions related to global existence and the regularity of solutions. It should also be noted that (3.2) gives a precise statement about the rate of regularization as $t$ approaches 0 .

(b) The formulation of Theorem 3.1(b) is inspired by [1, Proposition 6.1]. 


\section{Global existence}

In order to formulate our next result on global existence we need to introduce some more notation. Assume $J \subset \mathbb{R}^{+}$is an interval which contains 0 and is right open. If $\mu \in(0,1]$ and $E$ is a Banach space we set

$$
\begin{aligned}
& C_{1-\mu}(J, E):=\left\{v \in C(\dot{J}, E) ; v \in B U C_{1-\mu}([0, T], E), \quad T<\sup J\right\}, \\
& C_{1-\mu}^{1}(J, E):=\left\{v \in C^{1}(\dot{J}, E) ; v, \dot{v} \in C_{1-\mu}(J, E)\right\}
\end{aligned}
$$

and equip these spaces with the natural Fréchet topology induced by the topology of $B U C_{1-\mu}([0, T], E)$ and $B U C_{1-\mu}^{1}([0, T], E)$, respectively. We recall that in case $\mu=1$ we obtain

$$
C_{0}^{1}(J, E):=C^{1}(J, E), \quad C_{0}(J, E):=C(J, E),
$$

with the Fréchet topology of uniform convergence on compact subsets of $J$.

Let $u$ be a solution of (1.1) on an interval $J$. Then $u$ is called a maximal solution if there does not exist a solution $v$ on an interval $J^{\prime}$ strictly containing $J$ such that $\left.v\right|_{J}=u$. If $u$ is a maximal solution, $J$ is called the maximal interval of existence. Finally, $u$ is called a global solution if $J=\mathbb{R}^{+}$.

We are now ready to formulate our main result on global existence and uniqueness.

THEOREM 4.1. Let $\alpha \in(0,1)$ be fixed and let $E_{\alpha}:=\left(E_{0}, E_{1}\right)_{\alpha}$ be a continuous interpolation space. Assume that $V_{\alpha} \subset E_{\alpha}$ is open and that

$(A, f) \in C^{1-}\left(V_{\alpha}, \mathcal{M}_{\alpha}\left(E_{1}, E_{0}\right) \times E_{0}\right)$.

(a) The quasilinear evolution equation (1.1) has for each initial value $x \in V_{\alpha}$ a unique maximal solution

$u(\cdot, x) \in C_{1-\alpha}^{1}\left(J(x), E_{0}\right) \cap C_{1-\alpha}\left(J(x), E_{1}\right)$.

The maximal interval of existence $J(x)$ is open in $\mathbb{R}^{+}$. Moreover,

$u(\cdot, x) \in C\left(J(x), V_{\alpha}\right) \cap C^{\alpha-\beta}\left(J(x), E_{\beta}\right)$

for any $\beta \in[0, \alpha)$, where $E_{\beta}:=\left(E_{0}, E_{1}\right)_{\beta}$.

(b) Assume that $x \in V_{\alpha} \cap E_{\delta}$ for some $\delta \in(\alpha, 1]$. Then

$u(\cdot, x) \in C_{1-\delta}^{1}\left(J(x), E_{0}\right) \cap C_{1-\delta}\left(J(x), E_{1}\right) \cap C\left(J(x), E_{\delta}\right)$.

(c) Let $x \in V_{\alpha}$ be given and suppose that $t^{+}(x):=\sup J(x)<\infty$. Then the following alternatives hold:

- $\operatorname{dist}\left(u(t, x), \partial V_{\alpha}\right) \rightarrow 0$ as $t \rightarrow t^{+}(x)$, or

- $u(\cdot, x) \notin U C\left(\left[0, t^{+}(x)\right), E_{\alpha}\right)$. 
(d) Suppose that the embedding $E_{1} \hookrightarrow E_{0}$ is compact. Let $x \in V_{\alpha}$ be given and suppose that $t^{+}(x)<\infty$. Then either

- $\operatorname{dist}\left(u(t, x), \partial V_{\alpha}\right) \rightarrow 0$ as $t \rightarrow t^{+}(x)$, or

- $\|u(t, x)\|_{E_{\delta}} \rightarrow \infty$ for each $\delta \in(\alpha, 1]$ as $t \rightarrow t^{+}(x)$.

(e) Suppose that $E_{1} \hookrightarrow E_{0}$ is compact, that the orbit $\gamma(x)$ is bounded in $E_{\delta}$ for some $\delta>\alpha$ and bounded away from $\partial V_{\alpha}$. Then $t^{+}(x)=\infty$. If, in addition, $u(\cdot, x) \in$ $U C\left(\mathbb{R}^{+}, E_{\alpha}\right)$, then $\{u(t, x) ; t \geq \tau\}$ is bounded in $E_{1}$ for any $\tau>0$.

Proof. (a) (i) It follows from Theorem 3.1(a) that there exists a number $\tau_{1}$ such that the quasilinear Problem (1.1) has a unique solution

$$
u_{1} \in \mathbb{E}_{1}\left(J_{1}\right):=B U C_{1-\mu}^{1}\left(J_{1}, E_{0}\right) \cap B U C_{1-\mu}\left(J_{1}, E_{1}\right),
$$

where $J_{1}:=\left[0, \tau_{1}\right]$. Let $x_{1}:=u_{1}\left(\tau_{1}\right)$. Then $x_{1} \in V_{\alpha} \cap E_{1}$ and we can apply Theorem 3.1(a) once again to obtain a unique solution $u_{2} \in \mathbb{E}_{1}\left(J_{2}\right) \cap C\left(J_{2}, E_{\alpha}\right)$ of

$\dot{u}+A(u) u=f(u), \quad u(0)=x_{1}$,

where $J_{2}:=\left[0, \tau_{2}\right]$. Clearly, $u_{2}$ solves the inhomogeneous linear Cauchy problem

$\dot{u}+\bar{A}(t) u=\bar{f}(t), \quad t \in J_{2}, \quad u(0)=x_{1}$,

where $\bar{A}(t):=A\left(u_{2}(t)\right)$ and $\bar{f}(t):=f\left(u_{2}(t)\right)$ for $t \in J_{2}$. It follows from the mapping properties of $(A, f)$ that

$(\bar{A}, \bar{f}) \in B U C\left(J_{2}, \mathcal{L}\left(E_{1}, E_{0}\right) \times E_{0}\right)$.

In particular, note that $\bar{f} \in B U C\left(J_{2}, E_{0}\right) \subset \mathbb{E}_{0}\left(J_{2}\right)$. We infer from Lemma 2.8(a) that $u_{2}$ is the unique solution of (4.3) in $\mathbb{E}_{1}\left(J_{2}\right)$. On the other side, we obtain from Lemma 2.6 that $\bar{A}(s) \in \mathcal{M}_{1}\left(E_{1}, E_{0}\right)$ for $s \in J_{2}$, and Lemma 2.8(a) now yields

$v:=\left(\frac{d}{d t}+\bar{A}(\cdot), \gamma\right)^{-1}\left(\bar{f}, x_{1}\right) \in C^{1}\left(J_{2}, E_{0}\right) \cap C\left(J_{2}, E_{1}\right) \subset \mathbb{E}_{1}\left(J_{2}\right)$

due to the fact that $\left(\bar{f}, x_{1}\right) \in B U C\left(J_{2}, E_{0}\right) \times E_{1}$. By the uniqueness of solutions of (4.3) in $\mathbb{E}_{1}\left(J_{2}\right)$ we conclude that

$u_{2}=v \in C^{1}\left(J_{2}, E_{0}\right) \cap C\left(J_{2}, E_{1}\right)$.

Let

$u(t):= \begin{cases}u_{1}(t) & \text { for } 0 \leq t \leq \tau_{1}, \\ u_{2}\left(t-\tau_{1}\right) & \text { for } \tau_{1} \leq t \leq \tau_{1}+\tau_{2}\end{cases}$ 
and set $J=\left[0, \tau_{1}+\tau_{2}\right]$. If follows from (4.2) and (4.5) that $u \in B U C_{1-\mu}\left(J, E_{1}\right)$. Moreover, $\dot{u}_{1}\left(\tau_{1}\right)=-A\left(x_{1}\right) x_{1}+f\left(x_{1}\right)=\dot{u}_{2}(0)$ and thus $u \in B U C_{1-\mu}^{1}\left(J, E_{0}\right)$. We conclude from Lemma 2.2(d) that

$u \in B U C_{1-\mu}^{1}\left(J, E_{0}\right) \cap B U C_{1-\mu}\left(J, E_{1}\right) \cap B U C^{\alpha-\beta}\left(J, E_{\beta}\right)$

and that $u$ is a solution of (1.1) on $J$.

(ii) Let

$J(x):=\bigcup\{[0, \tau] ;(1.1)$ has a solution on $[0, \tau]\}$.

It is clear that $J(x)$ is right open, since we could otherwise repeat the steps of part (i) which contradicts the definition of $J(x)$. The construction in (i) shows that the maximal solution $u(\cdot, x)$ is unique and satisfies (4.1).

(b) Let $T \in \dot{J}(x)$ be arbitrary and set $I:=[0, T]$. It follows from step (a) that the maximal solution $u(\cdot, x)$ of $(1.1)$ satisfies

$u(\cdot, x) \in B U C_{1-\alpha}^{1}\left(I, E_{0}\right) \cap B U C_{1-\alpha}\left(I, E_{1}\right) \cap B U C\left(I, V_{\alpha}\right)$.

Let $\bar{A}(t):=A(u(t, x)), \bar{f}(t):=f(u(t, x))$ for $t \in I$. We conclude that

$(\bar{A}, \bar{f}) \in B U C\left(I, \mathcal{L}\left(E_{1}, E_{0}\right) \times E_{0}\right)$

and that $v:=\left.u(\cdot, x)\right|_{I}$ is the unique solution of the inhomogeneous linear problem

$\dot{v}+\bar{A}(t) v=\bar{f}(t), \quad t \in I, \quad v(0)=x$

in the set (4.6). Lemma 2.6, Lemma 2.8(a) and (4.7), on the other side, yield

$w:=\left(\frac{d}{d t}+\bar{A}(\cdot), \gamma\right)^{-1}(\bar{f}, x) \in B U C_{1-\delta}^{1}\left(I, E_{0}\right) \cap B U C_{1-\delta}\left(I, E_{1}\right)$

due to the fact that $(\bar{f}, x) \in B U C_{1-\delta}\left(I, E_{0}\right) \times E_{\delta}$. Since

$B U C_{1-\delta}^{1}\left(I, E_{0}\right) \cap B U C_{1-\delta}\left(I, E_{1}\right) \hookrightarrow B U C_{1-\alpha}^{1}\left(I, E_{0}\right) \cap B U C_{1-\alpha}\left(I, E_{1}\right)$

we conclude that $v=w$, that is, $v$ has the same regularity as $w$. Since $T \in J(x)$ can be chosen arbitrarily, the assertion follows from Lemma 2.2(b).

(c) We argue by contradiction and assume that $u \in U C\left(\left[0, t^{+}\right), E_{\alpha}\right)$, and that $\operatorname{dist}\left(u(t, x), \partial V_{\alpha}\right) \not \rightarrow 0$ as $t \rightarrow t^{+}$, where $t^{+}:=t^{+}(x)$. Let $S$ be the closure of $\left\{u(t, x) ; 0 \leq t<t^{+}\right\}$in $E_{\alpha}$. Since $u \in U C\left(\left[0, t^{+}\right), E_{\alpha}\right)$ we conclude that $S$ is compact in $V_{\alpha}$ and it follows from Theorem 3.1(b) that there exists a number $\tau=\tau(S)>0$ such that the quasilinear equation (1.1) has a unique solution

$v(\cdot, y) \in B U C_{1-\alpha}^{1}\left([0, \tau], E_{0}\right) \cap B U C_{1-\alpha}\left([0, \tau], E_{1}\right)$

on $[0, \tau]$ for any $y \in S$. We choose $t_{1} \in\left(0, t^{+}\right)$such that $t_{1}+\tau>t^{+}$and we set $x_{1}:=u\left(t_{1}, x\right)$ and $v_{1}:=v\left(\cdot, x_{1}\right)$. Since $x_{1} \in V_{\alpha} \cap E_{1}$ we infer from (4.8) and step (b) that $v_{1}$ enjoys the regularity property

$v_{1} \in C^{1}\left([0, \tau], E_{0}\right) \cap C\left([0, \tau], E_{1}\right)$. 
Let

$u(t):= \begin{cases}u(t, x) & \text { for } 0 \leq t \leq t_{1}, \\ v_{1}\left(t-t_{1}\right) & \text { for } t_{1} \leq t \leq t_{1}+\tau\end{cases}$

and set $I:=\left[0, t_{1}+\tau\right]$. We conclude that

$u \in B U C_{1-\alpha}^{1}\left(I, E_{0}\right) \cap B U C_{1-\alpha}\left(I, E_{1}\right)$,

and that $u$ is a solution of the quasilinear equation (1.1) with initial value $x$. Hence, $u$ is a proper extension of $u(\cdot, x)$, contradicting the maximality of $u(\cdot, x)$.

(d) Suppose the claim is not correct. Then there exists a number $\delta \in(\alpha, 1]$, numbers $r, R>0$, a set $U \subset V_{\alpha}$, and a sequence $\left(t_{k}\right)$ such that

- $\operatorname{dist}\left(U, \partial V_{\alpha}\right) \geq r$,

- $t_{k} \rightarrow t^{+}(x)$ as $k \rightarrow \infty$,

- $u\left(t_{k}, x\right) \in U \cap \mathbb{B}_{E_{\delta}}(0, R)$ for every $k \in \mathbb{N}$.

Let $S$ be the closure of $\left\{u\left(t_{k}, x\right) ; k \in \mathbb{N}\right\}$ in $E_{\alpha}$. It follows from the compact embedding $E_{\delta} \hookrightarrow E_{\alpha}$ that $S$ is a compact subset of $V_{\alpha}$. As in part (c) we conclude that the solution $u(\cdot, x)$ can be continued beyond $t^{+}(x)$, which leads to a contradiction.

(e) It follows from part (d) of the theorem that $t^{+}(x)=\infty$. Next, we infer from the compact embedding $E_{\delta} \hookrightarrow E_{\alpha}$ that $\gamma(x)$ is relatively compact in $V_{\alpha}$. Since locally Lipschitz continuous functions are uniformly Lipschitz continuous on compact sets we conclude that there exists a constant $L$ such that

$\left\|(A, f)\left(z_{1}\right)-(A, f)\left(z_{2}\right)\right\|_{\mathcal{L}\left(E_{1}, E_{0}\right) \times E_{0}} \leq L\left\|z_{1}-z_{2}\right\|_{E_{\alpha}}, \quad z_{1}, z_{2} \in \gamma(x)$.

In the sequel we set $(\bar{A}(t), \bar{f}(t)):=A(u(t, x)), f(u(t, x))$ for $t \in \mathbb{R}^{+}$. It is a consequence of (4.10) and our assumptions that

$(\bar{A}, \bar{f}) \in B U C\left(\mathbb{R}^{+}, \mathcal{L}\left(E_{1}, E_{0}\right) \times E_{0}\right)$.

We infer from Lemma 2.5(b) and Lemma 2.6 that there exists a constant $M \geq 1$ such that

$\left\|\left(\frac{d}{d t}+\bar{A}(s), \gamma\right)^{-1}\right\|_{\mathcal{L}\left(C\left(I, E_{0}\right) \times E_{1}, C^{1}\left(I, E_{0}\right) \cap C\left(I, E_{1}\right)\right)} \leq M, \quad s \in \mathbb{R}^{+}$,

where $I:=[0,1]$. It is then easy to see that

$\left\|K_{\omega+\bar{A}(s)}\right\|_{\mathcal{L}\left(C\left(I, E_{0}\right), C\left(I, E_{1}\right)\right)} \leq M, \quad\left\|e^{-t(\omega+\bar{A}(s))}\right\|_{\mathcal{L}\left(E_{1}\right)} \leq M e^{-w t}$

for any $t \in I, s \in \mathbb{R}^{+}$and any $\omega \geq 0$. Let $\eta:=\left(4 M^{2}\right)^{-1}$. It follows from (4.11) that there are numbers $T \in(0,1]$ and $\omega=\omega(T)>0$ such that

$M e^{-\omega T}<1 / 2, \quad\|\bar{A}(s+\tau)-\bar{A}(s)\|_{\mathcal{L}\left(E_{1}, E_{0}\right)} \leq \eta, \quad \tau \in[0, T], s \in \mathbb{R}^{+}$. 
Next we observe that $u:=u(\cdot, x)$ is the unique solution of

$$
\left(\frac{d}{d t}+\omega+\bar{A}(\cdot), \gamma\right) u=(\omega u+\bar{f}, x)=:(g, x) \quad \text { on } \mathbb{R}^{+} \text {. }
$$

Since $u(\tau) \in E_{1}$ for any $\tau>0$ we can, and will, assume that $x \in E_{1}$. Let $n \in \mathbb{N}^{*}$ be an arbitrary integer and let $v_{j}(t):=u(j T+t)$ for $t \in[0, T]$ and $j \in\{0, \ldots, n\}$. Then it is clear that $v_{j}$ is the unique solution of

$$
\left(\frac{d}{d t}+\omega+\bar{A}(j T+\cdot), \gamma\right) v=\left(g_{j}, u(j T)\right) \quad \text { on } I,
$$

where $g_{j}(t):=g(j T+t)$ for $t \in[0, T]$. It follows from (4.12)-(4.14) that $v_{j}$ admits the following representation:

$$
\begin{aligned}
& v_{j}=\left(\frac{d}{d t}+\omega+\bar{A}_{j}, \gamma\right)^{-1}\left[(\mathrm{id}, \mathrm{id})+\left(B_{j}(\cdot), 0\right)\right. \\
& \left.\left(\frac{d}{d t}+\omega+\bar{A}_{j}, \gamma\right)^{-1}\right]^{-1}\left(g_{j}, u(j T)\right) \\
& =K_{w+\bar{A}_{j}}\left[1+B_{j}(\cdot) K_{\omega+\bar{A}_{j}}\right]^{-1}\left(g_{j}-B_{j}(\cdot) e^{-\cdot\left(\omega+\bar{A}_{j}\right)}\right. \\
& u(j T))+e^{-\cdot\left(\omega+\bar{A}_{j}\right)} u(j T),
\end{aligned}
$$

where $\bar{A}_{j}:=\bar{A}(j T), B_{j}(\tau):=(\bar{A}(j T+\tau)-\bar{A}(j T))$ for $\tau \in[0, T]$ and where (id, id) $:=\left(\operatorname{id}_{C\left(I, E_{0}\right)}, \operatorname{id}_{E_{1}}\right)$. Thanks to (4.13) and (4.14) we have

$$
\left\|K_{w+\bar{A}_{j}}\left[1+B_{j}(\cdot) K_{\omega+\bar{A}_{j}}\right]^{-1}\right\|_{\mathcal{L}\left(C\left(I, E_{0}\right), C\left(I, E_{1}\right)\right)} \leq 2 M, \quad j \in\{0, \ldots, n\} .
$$

It follows now from the representation formula for $v_{j}$ and from (4.14) that $u(n T)=$ $v_{n-1}(T)$ satisfies the estimate

$$
\begin{aligned}
\|u(n T)\|_{E_{1}} & \leq 2 M\|g\|_{L_{\infty}\left(\mathbb{R}^{+}, E_{0}\right)}\left(1+q+\cdots q^{n-1}\right)+q^{n}\|x\|_{E_{1}} \\
& \leq 2 M(1-q)^{-1}\|g\|_{L_{\infty}\left(\mathbb{R}^{+}, E_{0}\right)}+\|x\|_{E_{1}}
\end{aligned}
$$

since $q:=\left(2 M^{2} \eta+M e^{-\omega T}\right)<1$. Let $t>0$ be arbitrary. Then we find a number $n \in \mathbb{N}$ and a number $\tau \in[0, T)$ such that $t=n T+\tau$. Observing that $u(t)=u(n T+\tau)=v_{n}(\tau)$ we can use the representation formula for $v_{n}$ and (4.15) to conclude that

$$
\begin{aligned}
\|u(t)\|_{E_{1}} & \leq 2 M\|g\|_{L_{\infty}\left(\mathbb{R}^{+}, E_{0}\right)}+\left(2 M^{2} \eta+M\right)\|u(n T)\|_{E_{1}} \\
& \leq c\left(\omega\|u\|_{L_{\infty}\left(\mathbb{R}^{+}, E_{0}\right)}+\|\bar{f}\|_{L_{\infty}\left(\mathbb{R}^{+}, E_{0}\right)}+\|x\|_{E_{1}}\right)
\end{aligned}
$$

with a universal constant $c$. 


\section{The semiflow property}

Let $X=(X, d)$ be a metric space and let $t^{+}: X \rightarrow(0, \infty]$ be a mapping. Then we define

$$
\mathcal{D}:=\bigcup_{x \in X}\left[0, t^{+}\right) \times\{x\}
$$

For a given map $\varphi: \mathcal{D} \rightarrow X$ we use the notation $\varphi^{t}(x):=\varphi(t, x)$. Then $\varphi$ is called a continuous (local) semiflow on $X$ if

- $\mathcal{D}$ is open in $\mathbb{R}^{+} \times V$,

- $\varphi \in C(\mathcal{D}, X)$,

- $\varphi^{0}=\mathrm{id}_{X}$,

- $\varphi^{s+t}(x)=\varphi^{t} \circ \varphi^{s}(x)$ whenever $0 \leq s<t^{+}(x)$ and $0 \leq t<t^{+}\left(\varphi^{s}(x)\right)$.

We write $\varphi \in C^{0,1-}(\mathcal{D}, X)$ if

- $\varphi \in C(\mathcal{D}, X)$,

- for every point $\left(t_{0}, x_{0}\right) \in \mathcal{D}$ there exist a product neighborhood $U \times V \subset \mathcal{D}$ and a constant $c>0$ such that

$$
d\left(\varphi^{t}(x), \varphi^{t}(y)\right) \leq c d(x, y), \quad(t, x),(t, y) \in U \times V .
$$

A semiflow $\varphi$ is called (locally) Lipschitz continuous if $\varphi \in C^{0,1-}(\mathcal{D}, X)$.

The following result shows that the quasilinear parabolic evolution equation (1.1) generates a locally Lipschitz continuous semiflow on $V_{\alpha}$.

THEOREM 5.1. Let $\alpha \in(0,1)$ be fixed and let $E_{\alpha}:=\left(E_{0}, E_{1}\right)_{\alpha}$ be a continuous interpolation space. Assume that $V_{\alpha} \subset E_{\alpha}$ is open and that

$$
(A, f) \in C^{1-}\left(V_{\alpha}, \mathcal{M}_{\alpha}\left(E_{1}, E_{0}\right) \times E_{0}\right) .
$$

Then $\mathcal{D}:=\bigcup_{x \in V_{\alpha}} J(x) \times\{x\}$ is open in $\mathbb{R}^{+} \times V_{\alpha}$ and

$$
[(t, x) \mapsto u(t, x)] \in C^{0,1-}\left(\mathcal{D}, V_{\alpha}\right) .
$$

The map $[(t, x) \mapsto u(t, x)]$ defines a locally Lipschitz continuous semiflow on $V_{\alpha}$.

Proof. Here we follow the arguments used in the proof of [1, Theorem 7.2]. Let $\left(t_{0}, x_{0}\right) \in \mathcal{D}$ be given and let $S:=\left\{u\left(t, x_{0}\right), 0 \leq t \leq t_{0}\right\}$. Since $u\left(\cdot, x_{0}\right) \in C\left(\left[0, t_{0}\right], V_{\alpha}\right)$ we conclude that $S$ is compact in $V_{\alpha}$. According to Theorem 3.1(b) there exist numbers $\varepsilon, \tau, L>0$ such that

- $\overline{\mathbb{B}}_{E_{\alpha}}(S, \varepsilon) \subset V_{\alpha}$,

- $u(\cdot, x)$ is defined on $[0, \tau]$ for each $x \in \overline{\mathbb{B}}_{E_{\alpha}}(S, \varepsilon)$,

- $\|u(s, x)-u(s, y)\|_{E_{\alpha}} \leq L\|x-y\|_{E_{\alpha}}$ for each $x, y \in \overline{\mathbb{B}}_{E_{\alpha}}(S, \varepsilon), s \in[0, \tau]$. 
Without loss of generality we can assume that $L \geq 1$. Next we fix numbers $\left\{\tau_{j} ; j=\right.$ $0, \ldots, m+1\}$ such that

$$
0=: \tau_{0}<\tau_{1}<\cdots \tau_{m}:=t_{0}, \quad \tau_{m+1}:=t_{0}+\tau
$$

and such that $\tau_{j+1}-\tau_{j} \leq \tau$ for $j=0, \ldots, m$. Finally we set $\varepsilon_{j}:=L^{j-m-1} \varepsilon$ for $j=0, \ldots, m+1$. Clearly we have $\varepsilon_{j} \leq \varepsilon$ and $\varepsilon_{j+1}=L \varepsilon_{j}$ for each $j$. It follows from (5.2) that $u(\cdot, x)$ exists on $\left[0, \tau_{j+1}-\tau_{j}\right]$ for each $x \in \overline{\mathbb{B}}_{E_{\alpha}}\left(u\left(\tau_{j}, x_{0}\right), \varepsilon_{j}\right)$ and $j=0, \ldots, m$. Moreover, we conclude from (5.2) that

$$
\begin{aligned}
& \qquad u(s, x) \in \overline{\mathbb{B}}_{E_{\alpha}}\left(u\left(s+\tau_{j}, x_{0}\right), \varepsilon_{j+1}\right), \quad s \in\left[0, \tau_{j+1}-\tau_{j}\right], \\
& \qquad\|u(s, x)-u(s, y)\|_{E_{\alpha}} \leq L\|x-y\|_{E_{\alpha}}, \quad s \in\left[0, \tau_{j+1}-\tau_{j}\right] \\
& \text { for each } x, y \in \overline{\mathbb{B}}_{E_{\alpha}}\left(u\left(\tau_{j}, x_{0}\right), \varepsilon_{j}\right) \text { and } j=0, \ldots, m .
\end{aligned}
$$

(i) We infer from the first line in $(5.3)$ that $\left[0, t_{0}+\tau\right] \subset J(x)$ and that

$$
u(t, x) \in \overline{\mathbb{B}}_{E_{\alpha}}(S, \varepsilon) \quad(t, x) \in\left[0, t_{0}+\tau\right] \times \overline{\mathbb{B}}_{E_{\alpha}}\left(x_{0}, \varepsilon_{0}\right) .
$$

Hence $\left[0, t_{0}+\tau\right] \times \overline{\mathbb{B}}_{E_{\alpha}}\left(x_{0}, \varepsilon_{0}\right) \subset \mathcal{D}$, showing that $\mathcal{D}$ is open in $\mathbb{R}^{+} \times V_{\alpha}$.

(ii) It follows from the second line of equation (5.3) that there exists a $c$ such that

$$
\begin{aligned}
& \|u(t, x)-u(t, y)\|_{E_{\alpha}} \leq c\|x-y\|_{E_{\alpha}}, \\
& \quad(t, x),(t, y) \in\left[0, t_{0}+\tau\right] \times \overline{\mathbb{B}}_{E_{\alpha}}\left(x_{0}, \varepsilon_{0}\right) .
\end{aligned}
$$

We have proved that the mapping $[x \mapsto u(t, x)]: \overline{\mathbb{B}}_{E_{\alpha}}\left(x_{0}, \varepsilon_{0}\right) \rightarrow V_{\alpha}$ is (globally) Lipschitz continuous, uniformly in $t \in\left[0, t_{0}+\tau\right]$. Moreover, it follows from (5.5) that

$\left\|u(t, x)-u\left(t_{0}, x_{0}\right)\right\|_{E_{\alpha}} \leq c\left\|x-x_{0}\right\|_{E_{\alpha}}+\left\|u\left(t, x_{0}\right)-u\left(t_{0}, x_{0}\right)\right\|_{E_{\alpha}}$

for $(t, x) \in\left[0, t_{0}+\tau\right] \times \overline{\mathbb{B}}_{E_{\alpha}}\left(x_{0}, \varepsilon_{0}\right)$. Since $u\left(\cdot, x_{0}\right) \in C\left(\left[0, t_{0}+\tau\right], E_{\alpha}\right)$ we conclude that the mapping $[(t, x) \mapsto u(t, x)]$ is continuous on $\mathcal{D}$. This and (5.5) shows (5.1).

(iii) The semiflow property in an immediate consequence of Theorem 4.1 and this completes the Proof of Theorem 5.1.

REMARKS 5.2. (a) Suppose that $\left(E_{0}, E_{1}\right)$ is a densely injected couple of Banach spaces and that $(\cdot, \cdot)_{\theta}$ is an admissible interpolation functor, that is, $(\cdot, \cdot)_{\theta}$ is for each $\theta \in(0,1)$ an interpolation functor of exponent $\theta$ such that $E_{1}$ is densely embedded in $E_{\theta}:=\left(E_{0}, E_{1}\right)_{\theta}$. Then the following result has been obtained in [2, Theorem 12.1]: 
THEOREM. (Amann) Suppose that $0<\gamma \leq \alpha<\sigma<1$, that $V_{\alpha}$ is open in $E_{\alpha}$, and

$(A, f) \in C^{1-}\left(V_{\alpha}, \mathcal{H}\left(E_{1}, E_{0}\right) \times E_{\gamma}\right)$.

Then Problem (1.1) has for each $x \in V_{\sigma}:=E_{\sigma} \cap V_{\alpha}$ a unique maximal solution

$u(\cdot, x) \in C^{1}\left(\dot{J}, E_{0}\right) \cap C\left(\dot{J}, E_{1}\right) \cap C\left(J, V_{\sigma}\right) \cap C^{\sigma}\left(J, E_{0}\right)$,

where $J=\left[0, t^{+}(x)\right)$, and where $V_{\sigma}$ is equipped with the topology of $E_{\sigma}$. Moreover, $[(t, x) \mapsto u(t, x)] \in C^{0,1-}\left(\mathcal{D}, V_{\sigma}\right)$.

Amann's result gives great flexibility for the choice of the interpolation spaces, and there are no geometric restrictions on the Banach spaces $E_{1}$ and $E_{0}$. Theorems 3.1, 4.1 and 5.1, on the other side, do require stringent geometric conditions on $E_{1}$ and $E_{0}$, subsumed in Definition 2.2, but provide sharper results: Here $\sigma=\alpha$ and $\gamma=0$ are admitted.

(b) In [4] local existence was proved for initial values in $V_{\sigma}$ for $\sigma>\alpha$ under the same assumptions as in the present paper. This result was extended in $[15,16]$ and it was shown that (1.1) generates a locally Lipschitz continuous semiflow on $V_{\sigma}$, again with $\sigma>\alpha$.

(c) Our results concerning global existence, see Theorem 4.1(c), (d), (e), are similar to the results stated in [3, Section 12].

(d) Under additional assumptions, the result of Amann stated in part (a) was extended in [9] to include situations were $E_{\sigma}$ is not necessarily an interpolation space between $E_{1}$ and $E_{0}$.

\section{Smoothness properties of solutions}

Let $\varphi: \mathcal{D} \rightarrow V_{\alpha}$ be the semiflow generated by the quasilinear evolution equation (1.1). Then we write $\varphi \in C^{0, k}\left(\mathcal{D}, V_{\alpha}\right)$ for $k \in \mathbb{N}^{*} \cup\{\infty, \omega\}$ if

- $\varphi(t, \cdot) \in C^{k}\left(\mathcal{D}_{t}, E_{\alpha}\right)$ for each $t \in \mathbb{R}^{+}$with $\mathcal{D}_{t}:=\left\{x \in V_{\alpha} ;(t, x) \in \mathcal{D}\right\} \neq \emptyset$,

- $D_{2}^{j} \varphi \in C\left(\mathcal{D}, E_{\alpha}\right)$ for $j=0, \ldots, k$,

and we call $\varphi$ a $C^{k}$-smooth semiflow.

THEOREM 6.1. Let $\alpha \in(0,1)$ be fixed and let $E_{\alpha}:=\left(E_{0}, E_{1}\right)_{\alpha}$ be a continuous interpolation space. Assume that $V_{\alpha} \subset E_{\alpha}$ is open and that

$$
(A, f) \in C^{k}\left(V_{\alpha}, \mathcal{M}_{\alpha}\left(E_{1}, E_{0}\right) \times E_{0}\right), \quad k \in \mathbb{N}^{*} \cup\{\infty, \omega\} .
$$

Then the maximal solution of the quasilinear evolution equation (1.1) satisfies

$$
u(\cdot, x) \in C^{k}\left(\dot{J}, E_{1}\right) \cap C^{k+1}\left(\dot{J}, E_{0}\right)
$$


and

$$
t^{m}\left(\frac{d}{d t}\right)^{m} u(\cdot, x) \in C_{1-\alpha}^{1}\left(J, E_{0}\right) \cap C_{1-\alpha}\left(J, E_{1}\right), \quad m=1, \ldots, k,
$$

where $J=\left[0, t^{+}(x)\right)$. Moreover,

$$
[(t, x) \mapsto u(t, x)] \in C^{0, k}\left(\mathcal{D}, V_{\alpha}\right) \cap C^{k}\left(\stackrel{o}{\mathcal{D}}, V_{\alpha}\right),
$$

where $\stackrel{o}{\mathcal{D}}:=\{(t, x) \in \mathcal{D} ; t>0\}$.

Proof. (i) Let $T \in\left(0, t^{+}(x)\right)$ be fixed and let $I:=[0, T]$. Then there exists a positive number $\delta>0$ such that $\lambda t \in\left[0, t^{+}(x)\right)$ for all $(\lambda, t) \in \Lambda \times I$, where $\Lambda:=$ $(1-\delta, 1+\delta)$. We set $u_{\lambda}(t):=u(\lambda t, x)$ for $(\lambda, t) \in \Lambda \times I$. It is easy to verify that $u_{\lambda} \in B U C_{1-\alpha}^{1}\left(I, E_{0}\right) \cap B U C_{1-\alpha}\left(I, E_{1}\right)$ and that $u_{\lambda}$ satisfies the parameterdependent evolution equation

$\left\{\begin{array}{l}\dot{v}+\lambda A(v) v=\lambda f(v) \\ v(0)=x\end{array}\right.$

on the interval $I$.

(ii) Let $i: B U C_{1-\alpha}^{1}\left(I, E_{0}\right) \cap B U C_{1-\alpha}\left(I, E_{1}\right) \mapsto B U C\left(I, E_{\alpha}\right)$ be the inclusion map of Lemma 2.2(b). Since $B U C\left(I, V_{\alpha}\right) \subset B U C\left(I, E_{\alpha}\right)$ is open we obtain that

$W_{\alpha}:=i^{-1}\left(B U C\left(I, V_{\alpha}\right)\right) \subset B U C_{1-\alpha}^{1}\left(I, E_{0}\right) \cap B U C_{1-\alpha}\left(I, E_{1}\right)=: \mathbb{E}_{1}(I)$

is open. We now define the mapping

$$
\begin{aligned}
\Phi: W_{\alpha} \times \Lambda \rightarrow B U C_{1-\alpha}\left(I, E_{0}\right) \times E_{\alpha} \\
(v, \lambda) \mapsto\left(\frac{d}{d t} v+\lambda A(v) v-\lambda f(v), \gamma v-x\right) .
\end{aligned}
$$

Observe that $\Phi\left(u_{\lambda}, \lambda\right)=0$ for $\lambda \in \Lambda$. It is well-known that the mapping properties of $(A, f)$ imply that

$$
(A, f) \in C^{k}\left(B U C\left(I, V_{\alpha}\right), B U C\left(I, \mathcal{L}\left(E_{1}, E_{0}\right)\right) \times B U C\left(I, E_{0}\right)\right) .
$$

Here we use the same notation for $(A, f)$ and the substitution operators induced by $(A, f)$. It is not difficult to see that

$[(B, v) \mapsto B v]: B U C\left(I, \mathcal{L}\left(E_{1}, E_{0}\right)\right) \times B U C_{1-\alpha}\left(I, E_{1}\right) \rightarrow B U C_{1-\alpha}\left(I, E_{0}\right)$

is continuous and bilinear, and thus real analytic. We conclude that

$\Phi \in C^{k}\left(W_{\alpha} \times \Lambda, B U C_{1-\alpha}\left(I, E_{0}\right) \times E_{\alpha}\right)$. 
(iii) Let $D_{1} \Phi$ denote the Fréchet derivative of $\Phi$ with respect to the first variable. Then

$$
D_{1} \Phi\left(u_{1}, 1\right) w=\left(\frac{d}{d t} w+A\left(u_{1}\right) w+\left(A^{\prime}\left(u_{1}\right) w\right) u_{1}+f^{\prime}\left(u_{1}\right) w, \gamma w\right) .
$$

It follows from (6.1) that the Fréchet derivative $\left(A^{\prime}, f^{\prime}\right)$ of $(A, f)$ satisfies

$$
\left(A^{\prime}, f^{\prime}\right) \in C\left(V_{\alpha}, \mathcal{L}\left(E_{\alpha}, \mathcal{L}\left(E_{1}, E_{0}\right) \times E_{0}\right)\right) .
$$

Let $B(t):=A\left(u_{1}(t)\right)+f^{\prime}\left(u_{1}(t)\right)$ for $t \in I$. Moreover, given any $z \in E_{\alpha}$ we set $R(t) z:=\left(A^{\prime}\left(u_{1}(t)\right) z\right) u_{1}(t)$ for $t \in \dot{I}$. It follows from (6.8) and Lemma 2.7(c) that

$$
\begin{aligned}
& B \in B U C\left(I, \mathcal{L}\left(E_{1}, E_{0}\right)\right), \quad B(s) \in \mathcal{M}_{\alpha}\left(E_{1}, E_{0}\right), \quad s \in I, \\
& \quad R \in B U C_{1-\alpha}\left(I, \mathcal{L}\left(E_{\alpha}, E_{0}\right)\right), \quad\|R\|_{B U C_{1-\alpha}\left(J_{\tau}, \mathcal{L}\left(E_{\alpha}, E_{0}\right)\right)} \leq N\left\|u_{1}\right\|_{\mathbb{E}_{1}\left(J_{\tau}\right)}
\end{aligned}
$$

where the constant $N$ is independent of $J_{\tau}:=[0, \tau] \subset I$. We conclude from (6.7), (6.9), and Lemma 2.8(b) that

$D_{1} \Phi\left(u_{1}, 1\right) \in \operatorname{Isom}\left(\mathbb{E}_{1}(I), \mathbb{E}_{0}(I) \times E_{\alpha}\right)$.

It follows from the Implicit Function Theorem that the equation $\Phi(v, \lambda)=0$ can be solved uniquely in terms of $\lambda$ in a neighborhood of 1 . Since $\Phi\left(u_{\lambda}, \lambda\right)=0$ we conclude from (6.6), (6.10) and the Implicit Function Theorem that there exists a number $\varepsilon \in(0, \delta)$ such that

$\left[\lambda \mapsto u_{\lambda}\right] \in C^{k}\left((1-\varepsilon, 1+\varepsilon), \mathbb{E}_{1}(I)\right)$.

Let $t_{0} \in \dot{I}$ be fixed. Given $u \in \mathbb{E}_{1}(I)$ we define $e(u):=u\left(t_{0}\right)$. It is easy to verify that $e \in \mathcal{L}\left(\mathbb{E}_{1}(I), E_{1}\right)$ and it follows from (6.11) that

$\left[\lambda \mapsto u_{\lambda}\left(t_{0}\right)=u_{1}\left(\lambda t_{0}\right)\right] \in C^{k}\left((1-\varepsilon, 1+\varepsilon), E_{1}\right)$.

Since this is true for any number $t_{0} \in \dot{I}$ we conclude that $u_{1} \in C^{k}\left(\dot{I}, E_{1}\right)$. It is now easy to see that

$$
\left.\left(\frac{d}{d \lambda}\right)^{m} u_{\lambda}\right|_{\lambda=1}=t^{m}\left(\frac{d}{d t}\right)^{m} u_{1} \in \mathbb{E}_{1}(I), \quad m=1, \ldots, k
$$

Since this is true for any interval $I=[0, T] \subset\left[0, t^{+}(x)\right)$ we have proved the first part of Theorem 6.1.

(iv) We now prove the first assertion in (6.3). Let $x_{0} \in V_{\alpha}$ and $t_{0} \in\left[0, t^{+}\left(x_{0}\right)\right)$ be fixed. Moreover, given $T \in\left(t_{0}, t^{+}\left(x_{0}\right)\right)$ let $I:=[0, T]$. It follows from Theorem 5.1 
that there exists a number $\delta>0$ such that $t^{+}(x) \geq T$ for all $x \in \mathbb{B}_{E_{\alpha}}\left(x_{0}, \delta\right)$. We consider the mapping

$$
\begin{gathered}
\Phi: W_{\alpha} \times \mathbb{B}_{E_{\alpha}}\left(x_{0}, \delta\right) \rightarrow B U C_{1-\alpha}\left(I, E_{0}\right) \times E_{\alpha} \\
(v, x) \mapsto\left(\frac{d}{d t} v+A(v) v-f(v), \gamma v-x\right),
\end{gathered}
$$

where $W_{\alpha}$ is defined in (ii). Let $u_{1}(\cdot, x):=\left.u(\cdot, x)\right|_{I}$ and note that $\Phi\left(u_{1}(\cdot, x), x\right)=0$ for $x \in \mathbb{B}_{E_{\alpha}}\left(x_{0}, \delta\right)$. As in (ii) we obtain that $\Phi$ is $C^{k}$-smooth. Moreover,

$D_{1} \Phi\left(u_{1}, x_{0}\right) w=\left(\frac{d}{d t} w+A\left(u_{1}\right) w+\left(A^{\prime}\left(u_{1}\right) w\right) u_{1}+f^{\prime}\left(u_{1}\right) w, \gamma w\right)$,

where we set $u_{1}:=u_{1}\left(\cdot, x_{0}\right)$. We conclude as in step (iii) that $D_{1} \Phi\left(u_{1}, x_{0}\right)$ is an isomorphism. The Implicit Function Theorem then implies that there exists a number $\varepsilon \in(0, \delta)$ such that

$\left[x \mapsto u_{1}(\cdot, x)\right] \in C^{k}\left(\mathbb{B}_{E_{\alpha}}\left(x_{0}, \varepsilon\right), \mathbb{E}_{1}(I)\right)$.

Given $u \in B U C\left(I, E_{\alpha}\right)$ we set $e(u):=u\left(t_{0}\right)$. Then $e \in \mathcal{L}\left(B U C\left(I, E_{\alpha}\right), E_{\alpha}\right)$ and we conclude from (6.13) and Lemma 2.2(b) that the mapping

$\mathbb{B}_{E_{\alpha}}\left(x_{0}, \varepsilon\right) \stackrel{C^{k}}{\rightarrow} \mathbb{E}_{1}(I) \stackrel{i}{\rightarrow} B U C\left(I, E_{\alpha}\right) \stackrel{e}{\rightarrow} E_{\alpha}, \quad x \mapsto u_{1}\left(t_{0}, x\right)$

is $C^{k}$-smooth. We have proved that $[(t, x) \mapsto u(t, x)] \in C^{0, k}\left(\mathcal{D}, V_{\alpha}\right)$.

(v) Let $x_{0} \in V_{\alpha}$ and $t_{0} \in\left[0, t^{+}\left(x_{0}\right)\right)$ be fixed. Given $T_{1} * \in\left(t_{0}, t^{+}\left(x_{0}\right)\right)$ there exists a number $\delta>0$ such that that $t^{+}(x) \geq T_{1} *$ for all $x \in \mathbb{B}_{E_{\alpha}}\left(x_{0}, \delta\right)$. We now choose $T \in\left(t_{0}, T_{1}\right)$ and set $I=[0, T]$. By making $\delta$ smaller if necessary we conclude that $\lambda t \in\left[0, T_{1}\right]$ for $(\lambda, t) \in \Lambda \times I$. Given $(\lambda, x) \in \Lambda \times \mathbb{B}_{E_{\alpha}}\left(x_{0}, \delta\right)$ we set $u_{\lambda}(\cdot, x):=\left.u(\lambda \cdot x)\right|_{I}$. Moreover, we define the mapping

$$
\begin{gathered}
\Phi: W_{\alpha} \times \Lambda \times \mathbb{B}_{E_{\alpha}}\left(x_{0}, \delta\right) \rightarrow B U C_{1-\alpha}\left(I, E_{0}\right) \times E_{\alpha} \\
(v,(\lambda, x)) \mapsto\left(\frac{d}{d t} v+\lambda A(v) v-\lambda f(v), \gamma v-x\right) .
\end{gathered}
$$

It follows that $\Phi\left(u_{\lambda}(\cdot, x),(\lambda, x)\right)=0$ for $(\lambda, x) \in \Lambda \times \mathbb{B}_{E_{\alpha}}\left(x_{0}, \delta\right)$. As before, we conclude that the mapping $\Phi$ is $C^{k}$-smooth and that the Fréchet derivative with respect to the first variable is

$$
D_{1} \Phi\left(u_{1},\left(1, x_{0}\right)\right) w=\left(\frac{d}{d t} w+A\left(u_{1}\right) w+\left(A^{\prime}\left(u_{1}\right) w\right) u_{1}+f^{\prime}\left(u_{1}\right) w, \gamma w\right) .
$$

The same arguments as above show that there exists a number $\varepsilon \in(0, \delta)$ such that

$$
\left[(\lambda, x) \mapsto u_{\lambda}(\cdot, x)\right] \in C^{k}\left((1-\varepsilon, 1+\varepsilon) \times \mathbb{B}_{E_{\alpha}}\left(x_{0}, \varepsilon\right), \mathbb{E}_{1}(I)\right) .
$$


Given $u \in B U C\left(I, E_{\alpha}\right)$ let $e(u):=u\left(t_{0}\right)$. Then $e \in \mathcal{L}\left(B U C\left(I, E_{\alpha}\right), E_{\alpha}\right)$ and it follows that

$$
\begin{aligned}
& (1-\varepsilon, 1+\varepsilon) \times \mathbb{B}_{E_{\alpha}}\left(x_{0}, \varepsilon\right) \stackrel{C^{k}}{\rightarrow} \mathbb{E}_{1}(I) \stackrel{i}{\rightarrow} B U C\left(I, E_{\alpha}\right) \stackrel{e}{\rightarrow} E_{\alpha}, \\
& (\lambda, x) \mapsto u_{\lambda}\left(t_{0}, x\right)=u_{1}\left(\lambda t_{0}, x\right)
\end{aligned}
$$

is $C^{k}$-smooth (for instance real analytic if $k=\omega$ ). We can now infer that

$[(t, x) \mapsto u(t, x)] \in C^{k}\left(\left(t_{0}-\varepsilon_{0}, t_{0}+\varepsilon_{0}\right) \times \mathbb{B}_{E_{\alpha}}\left(x_{0}, \varepsilon\right), V_{\alpha}\right)$

where $\varepsilon_{0}:=t_{0} \varepsilon$. We have proved that $[(t, x) \mapsto u(t, x)] \in C^{k}\left(\stackrel{o}{\mathcal{D}}, V_{\alpha}\right)$.

REMARKS 6.2. (a) Under stronger assumptions, smoothness properties of solutions were also considered in [1].

(b) Theorem 6.1 improves on the results contained in [16], where it was shown that (1.1) generates a smooth semiflow in the (stronger) topology of $E_{\sigma}$ with $\sigma>\alpha$.

(c) The idea to use the Implicit Function Theorem in the Proof of Theorem 6.1 is inspired by [4].

\section{Acknowledgment}

The authors would like to thank Jan Prüss for fruitful discussions and in particular for the Proof of Lemma 2.6.

\section{REFERENCES}

[1] Amann, H., Dynamic theory of quasilinear parabolic equations I. Abstract evolution equations, Nonlinear Anal. 12 (1993), 895-919.

[2] AmanN, H., Nonhomogeneous linear and quasilinear elliptic and parabolic boundary value problems, Function spaces, Differential Operators and Nonlinear Analysis (H. J. Schmeisser, H. Triebel, eds.) 9-126, Teubner-Texte Math., 133, Teubner, Stuttgart, 1993.

[3] Amann, H., Linear and Quasilinear Parabolic Problems, vol. I, Birkhäuser, Basel, 1995. vol. II, III, in preparation.

[4] Angenent, S., Nonlinear analytic semiflows, Proc. Roy. Soc. Edinburgh Sect. A 115 (1990), 91-107.

[5] BAILlON, J. B., Charactère borné de certains genérateurs de semigroupes linéaires dans les espaces de Banach, C.R. Acad. Sc. Paris 290 (1980), 757-760.

[6] CLÉMENT, PH. and LI, S., Abstract parabolic quasilinear equations and application to a groundwater flow problem, Adv. Math. Sci. Appl 3 (1993/94), Special Issue, 17-32.

[7] DA PrATO, G. and GRISVARD, P., Équations d'évolution abstraites nonlinéaires de type parabolique, Ann. Mat. Pura Appl.(4) 120 (1979), 329-396.

[8] DA Prato, G. and LunARDI, A., Stability, instability and center manifold theorem for fully nonlinear autonomous parabolic equations in Banach space, Arch. Rational Mech. Anal. 101 (1988), 115-144.

[9] EsCHER, J., On quasilinear fully parabolic boundary value problems, Differential Integral Equations 7 (1994), 1325-1343. 
[10] LUNARDI, A., Abstract quasilinear parabolic equations, Math. Ann. 267 (1984), 395-415.

[11] LUNARDI, A., Global solutions of abstract quasilinear parabolic equations, J. Differential Equations 58 (1985), 228-242.

[12] LUNARDI, A., Interpolation spaces between domains of elliptic operators and spaces of continuous functions with applications to nonlinear parabolic equations, Math. Nachr. 121 (1985), 295-318.

[13] LunARDI, A., Analytic Semigroups and Optimal Regularity in Parabolic Problems, Birkhäuser, Basel, 1995.

[14] PaZY, A., Semigroups of Linear Operators and Applications to Partial Differential Equations, Springer Verlag, New York, 1983.

[15] SimONETT, G., Zentrumsmannigfaltigkeiten für Quasilineare parabolische Gleichungen, Ph.D. thesis, Universität Zürich, 1992, and Institut für angewandte Analysis and Stochastik, Report Nr. 2, Berlin, 1992.

[16] SimonetT, G., Quasilinear parabolic equations and semiflows. Evolution Equations, Control Theory, and Biomathematics, 523-536, Lecture Notes in Pure and Appl. Math., 155, Dekker, New York, 1994.

[17] SimONETT, G., Center manifolds for quasilinear reaction-diffusion systems, Differential Integral Equations 8 (1995), 753-796

[18] SobolEVSKII, P. E., Equations of parabolic type, Amer. Math. Soc. Transl., Ser. 2, 49 (1966), 1-62.

[19] YAGI, A., Abstract quasilinear evolution equations of parabolic type in Banach spaces, Boll. UMI, 5-B (1991), 341-368.

\author{
Philippe Clément \\ Department of Mathematics and Informatics \\ Delft University of Technology \\ NL-2600 GA Delft \\ The Netherlands \\ e-mail: clement@twi.tudelft.nl \\ Gieri Simonett \\ Department of Mathematics \\ Vanderbilt University \\ Nashville, TN 37240 \\ USA \\ e-mail: simonett@math.vanderbilt.edu
}

To access this journal online:
http://www.birkhauser.ch 